\title{
On families of Lindelöf and related subspaces of $2^{\omega_{1}}$
}

\author{
by \\ Lúcia Junqueira and Piotr Koszmider (São Paulo)
}

\begin{abstract}
We consider the families of all subspaces of size $\omega_{1}$ of $2^{\omega_{1}}$ (or of a compact zero-dimensional space $X$ of weight $\omega_{1}$ in general) which are normal, have the Lindelöf property or are closed under limits of convergent $\omega_{1}$-sequences. Various relations among these families modulo the club filter in $[X]^{\omega_{1}}$ are shown to be consistently possible. One of the main tools is dealing with a subspace of the form $X \cap M$ for an elementary submodel $M$ of size $\omega_{1}$. Various results with this flavor are obtained. Another tool used is forcing and in this case various preservation or nonpreservation results of topological and combinatorial properties are proved. In particular we prove that there may be no c.c.c. forcing which destroys the Lindelöf property of compact spaces, answering a question of Juhász. Many related questions are formulated.
\end{abstract}

1. Introduction. The results of this paper are related to several closely located topics. First, one can draw a connection with the general topic of reflection. A classical theorem in logic, called the lower Löwenheim-Skolem theorem, states that for any infinite mathematical structure and any infinite cardinal $\kappa$, smaller than the cardinality of the structure, there is a substructure of cardinality $\kappa$ which satisfies the same first order formulas as the entire structure. For instance, every uncountable field has a subfield of cardinality $\omega_{1}$ with the same first order properties as the entire field. Most topological and many other properties cannot be expressed by first order formulas, which makes the problem of reflection of these properties to smaller substructures more subtle. What we consider a small or a large substructure is a fundamental factor determining the flavor of the methods of research and the results. For example, here we consider small or large in the sense of infinite cardinality, and thus set-theoretic methods are central. Many deep results with this flavor have been obtained. Probably the most famous one is Shelah's compactness theorem for certain algebraic structures of singular

2000 Mathematics Subject Classification: 03E35, 03E55, 54D20, 54A35.

The first author was partially supported by FAPESP (Research Foundation of the State of São Paulo) grant No. 1997/9813-0.

The second author was supported by FAPESP grant No. 1997/10491-7. 
sizes (see [23]). But there are also well known topological results of this sort, for example: if a compact first countable space is nonmetrizable, then it has a nonmetrizable subspace of size $\omega_{1}$ (Dow [6]); or if a regular space does not have a countable base, then it has a subspace of size $\omega_{1}$ without a countable base (Tkachenko [28], see also [16]).

In this paper we focus on the following three properties: normality, the Lindelöf property, and the property of being closed under limits of $\omega_{1}$ sequences which are convergent. We are focusing on the basic compact space $2^{\omega_{1}}$ but we make some detours into compact spaces in general or with some extra properties.

For a cardinal $\kappa$ and a topological space $X$ of cardinality greater than $\kappa$ we define the following subfamilies of $[X]^{\kappa}$ :

$$
\begin{aligned}
{[X]_{L}^{\kappa} } & =\left\{Y \in[X]^{\kappa}: Y \text { has the Lindelöf property }\right\} \\
{[X]_{N}^{\kappa} } & =\left\{Y \in[X]^{\kappa}: Y \text { is normal }\right\} \\
{[X]_{0}^{\kappa} } & =\left\{Y \in[X]^{\kappa}: Y \text { is closed under limits of } \omega_{1} \text {-sequences }\right\} .
\end{aligned}
$$

The main focus of this paper is to investigate how large the above families can be and how they are interrelated. To measure the size of the above families one may consider the relations between $[X]_{L}^{\omega_{1}},[X]_{N}^{\omega_{1}}$ and $[X]_{\circ}^{\omega_{1}}$ in the usual Boolean algebra of subsets of a compact space $X$. For example we always (i.e., for regular spaces) have $[X]_{L}^{\omega_{1}} \subseteq[X]_{N}^{\omega_{1}},[X]_{0}^{\omega_{1}}$ and for metrizable compact spaces we have $[X]_{L}^{\omega_{1}}=[X]_{N}^{\omega_{1}}=[X]_{\circ}^{\omega_{1}}=[X]^{\omega_{1}}$. For $X=2^{\omega_{1}}$ the finite Boolean algebra generated in $\wp\left(\wp\left(2^{\omega_{1}}\right)\right)$ by the families $\left[2^{\omega_{1}}\right]_{L}^{\omega_{1}},\left[2^{\omega_{1}}\right]_{N}^{\omega_{1}}$ and $\left[2^{\omega_{1}}\right]_{0}^{\omega_{1}}$ is completely determined in ZFC as shown in the following fact.

FACT 1.1. The following statements hold:

(a) $\left[2^{\omega_{1}}\right]_{N}^{\omega_{1}} \backslash\left[2^{\omega_{1}}\right]_{L}^{\omega_{1}} \neq \emptyset$;

(b) $\left[2^{\omega_{1}}\right]_{N}^{\omega_{1}} \backslash\left[2^{\omega_{1}}\right]_{0}^{\omega_{1}} \neq \emptyset$;

(c) $\left[2^{\omega_{1}}\right]_{0}^{\omega_{1}} \backslash\left[2^{\omega_{1}}\right]_{L}^{\omega_{1}} \neq \emptyset$;

(d) $\left[2^{\omega_{1}}\right]_{0}^{\omega_{1}} \backslash\left[2^{\omega_{1}}\right]_{N}^{\omega_{1}} \neq \emptyset$;

(e) $\emptyset \neq\left[2^{\omega_{1}}\right]_{L}^{\omega_{1}}$;

(f) $\left[2^{\omega_{1}}\right]_{0}^{\omega_{1}} \cup\left[2^{\omega_{1}}\right]_{N}^{\omega_{1}} \neq\left[2^{\omega_{1}}\right]^{\omega_{1}}$;

(g) $\left(\left[2^{\omega_{1}}\right]_{N}^{\omega_{1}} \cap\left[2^{\omega_{1}}\right]_{0}^{\omega_{1}}\right) \backslash\left[2^{\omega_{1}}\right]_{L}^{\omega_{1}} \neq \emptyset$.

Proof. It is clear that (b) implies (a) and (d) implies (c). To see (b), we define $f_{\alpha} \in 2^{\omega_{1}}$ for $\alpha<\omega_{1}$ by $f_{\alpha}(\beta)=0$ if $\beta \neq \alpha$ and $f_{\alpha}(\alpha)=1$. Then $Y=\left\{f_{\alpha}: \alpha<\omega_{1}\right\}$ witnesses that $\left[2^{\omega_{1}}\right]_{N}^{\omega_{1}} \backslash\left[2^{\omega_{1}}\right]_{0}^{\omega_{1}} \neq \emptyset$.

We now prove (d). The proof is a version of the proof of Stone's theorem that $\omega^{\omega_{1}}$ is not normal. In this version we use an Aronszajn tree. It is well known that if $\left\{e_{\alpha}: \alpha<\omega_{1}\right\}$ are such that $e_{\alpha}: \alpha \rightarrow \omega$ is a one-to-one mapping, $e_{\alpha}\left\lceil\beta={ }^{*} e_{\beta}\right.$ for $\beta<\alpha$, then $T^{\prime}=\left\{e_{\alpha}\lceil\beta: \beta \leq \alpha\}\right.$ is an Aronszajn tree as a subtree of $\omega^{<\omega_{1}}$ ([20], Theorem 5.9). Construct $\left(f_{\alpha}\right)_{\alpha<\omega_{1}}$ similarly, 
by induction, such that $f_{\alpha}: \omega \cdot \alpha \rightarrow 2$, and for every $\beta<\alpha$ there is $e_{\alpha}(\beta)$ such that $f_{\alpha}(\omega \cdot \beta+n)=1$ if $n<e_{\alpha}(\beta)$ and $f_{\alpha}(\omega \cdot \beta+n)=0$ if $n \geq e_{\alpha}(\beta)$, for $n \in \omega$ (here, we are really embedding $\omega^{\omega_{1}}$ into $2^{\omega_{1}}$ ). By the properties of the family $\left\{e_{\alpha}: \alpha<\omega_{1}\right\}$ mentioned above and by the construction of the family $\left\{f_{\alpha}: \alpha<\omega_{1}\right\}$, we see that $T=\left\{f_{\alpha}\lceil\beta: \beta \leq \omega \cdot \alpha\}\right.$ is an Aronszajn tree as a subtree of $2^{<\omega_{1}}$. It is also clear that $T^{*}$ is an Aronszajn tree, where $T^{*}$ denotes the set of all finite modifications of elements of $T$. Now we define

$$
X_{T}=\left\{t^{\frown} i \in 2^{\omega_{1}}: t \in T \text { and } i \in\{0,1\}\right\},
$$

where $t^{\frown} i$ for $i=0,1$ are given by $t^{\frown} 0(\alpha)=t(\alpha)$ if $\alpha \in \operatorname{dom}(t)$ and $t^{\frown} 0(\alpha)=$ 0 otherwise; $t^{\frown} 1(\alpha)=t(\alpha)$ if $\alpha \in \operatorname{dom}(t)$ and $t^{\frown} 1(\alpha)=1$ if $\alpha$ is a limit ordinal and $\alpha \notin \operatorname{dom}(t)$ and $t^{\curvearrowright} 1(\alpha)=0$ if $\alpha$ is a successor ordinal and $\alpha \notin \operatorname{dom}(t)$. We consider $X_{T}^{*} \subseteq 2^{\omega_{1}}$, the set of all finite modifications of the functions from $X_{T} \subseteq 2^{\omega_{1}}$. We will first show that $X_{T}^{*} \in\left[2^{\omega_{1}}\right]_{0}^{\omega_{1}}$ by showing that there are no (nontrivial) converging $\omega_{1}$-sequences with terms in $X_{T}^{*}$. Let $\left\{x_{\xi}: \xi<\omega_{1}\right\}$ be such that $x_{\xi} \in X_{T}^{*}$ and $x_{\xi} \neq x_{\xi^{\prime}}$ if $\xi \neq \xi^{\prime}$. Suppose $x_{\xi}=\left(t_{\xi} i\right)^{*}$, where $i \in\{0,1\}$ and $*$ denotes some finite modification. Since the levels of the tree are countable, we can assume that $t_{\xi} \in \operatorname{Lev}_{\alpha_{\xi}}(T)$ and $\alpha_{\xi}<\alpha_{\xi^{\prime}}$ for $\xi<\xi^{\prime}$. If $x$ is an $\omega_{1}$-limit of the $\omega_{1}$-sequence $\left\{x_{\xi}: \xi<\omega_{1}\right\}$, then for every $\alpha<\omega_{1}$, there is $\xi<\omega_{1}$ such that $\alpha_{\xi}>\alpha$ and $x\left\lceil\alpha=x_{\xi}\lceil\alpha\right.$. But $x_{\xi}\left\lceil\alpha\right.$ is a finite modification of $t_{\xi}\left\lceil\alpha\right.$, and thus $x$ is a branch of $T^{*}$, a contradiction.

We now have to show that $X_{T}^{*} \notin\left[2^{\omega_{1}}\right]_{N}^{\omega_{1}}$. We consider the sets

$$
F_{i}=\left\{t^{\frown} i \in 2^{\omega_{1}}: t \in T\right\},
$$

for $i=0,1$ and apply Lemma 2.3 from Section 2 . We have considered $X_{T}^{*}$ instead of $X_{T}$ because $X_{T}^{*}$ is a dense subspace of $2^{\omega_{1}}$. By Lemma 2.3, it is enough to show that $F_{0}$ and $F_{1}$ have disjoint closures in $X_{T}^{*}$. For this we note that for every $x \in X_{T}^{*}$, there is an open set $V$ such that $x \in V$ and there is $i \in$ $\{0,1\}$ satisfying $V \cap F_{i}=\emptyset$; indeed, let $x \in X_{T}^{*}$; then $x=\left(t^{\wedge} i\right)^{*}$ for some $t \in T$ and $i \in\{0,1\}$. We can pick $\beta<\omega_{1}$ such that $V_{0}=[(\omega \cdot \beta, 0),(\omega \cdot(\beta+1), 0)]$ or $V_{1}=[(\omega \cdot \beta, 1),(\omega \cdot \beta+1,0),(\omega \cdot(\beta+1), 1),(\omega \cdot(\beta+1)+1,0)]$ is an open neighborhood of $x$. Since each $e_{\xi}$ is a one-to-one mapping, we see that $V_{i} \cap F_{1-i}=\emptyset$, and we are done.

The clause (e) is witnessed by an $\omega_{1}$-convergent sequence with a limit, (f) can be witnessed by the disjoint union of a subspace witnessing (d) with a subspace witnessing (b). Finally for (g), we find a discrete subspace which has no converging $\omega_{1}$-subsequence. Consider $e_{\alpha}$ 's and $f_{\alpha}$ 's as before. Take $m>0$ such that the set $A=\left\{\beta<\omega_{1}: a_{\beta+1}(\beta)=m\right\}$ is uncountable, and then consider $x_{\beta}=f_{\beta+1} \bumpeq 0$. Then $\left\{x_{\beta}: \beta \in A\right\}$ is a discrete space and the proof of the fact that it has no convergent $\omega_{1}$-sequence is similar to the proof of the same property for $X_{T}^{*}$ in $(\mathrm{d})$. 
The best known open problem related here is whether $[X]_{L}^{\omega_{1}}$ is nonempty for all compact uncountable $X$ (see for example [12]).

In this paper we will consider the above families $[X]_{L}^{\omega_{1}},[X]_{N}^{\omega_{1}},[X]_{0}^{\omega_{1}}$ in the Boolean algebra of subsets of $X$ modulo the nonstationary ideal (or the club filter) in $[X]^{\omega_{1}}$ (this was first investigated by Jech [13] and Kueker [19]; see [3] for an introduction). Thus $\equiv$ will mean club equivalence, i.e., $A$ and $B$ are club equivalent if and only if there is a club $C \subseteq[X]^{\omega_{1}}$ such that $C \cap A=C \cap B$. Also, 0,1 and $\leq$ will denote respectively the zero, unit and Boolean order of the algebra. This approach turned out to be natural and fruitful in several contexts. For instance, Shelah bases the main results of $[26]$ on a lemma which states that $[X]_{L}^{\omega_{1}}$ is co-stationary if $X$ is Lindelöf with points $G_{\delta}$ (also [27] uses it in a fundamental way); and Dow uses the property of being or not a stationary subset of the set $\left\{Y \in[X]^{\omega}: \operatorname{cl}(Y)=Y\right\}$ to obtain reflection or nonreflection of metrizability $([7])$. Our results in this paper show that:

(a) $0 \leq\left[2^{\omega_{1}}\right]_{L}^{\omega_{1}} \equiv\left[2^{\omega_{1}}\right]_{N}^{\omega_{1}} \leq\left[2^{\omega_{1}}\right]_{\circ}^{\omega_{1}}<1$ (Corollary 2.9).

(b) It is consistent that $0 \equiv\left[2^{\omega_{1}}\right]_{L}^{\omega_{1}} \equiv\left[2^{\omega_{1}}\right]_{N}^{\omega_{1}} \equiv\left[2^{\omega_{1}}\right]_{0}^{\omega_{1}}<1$ (Corollary 3.4).

(c) Assuming the existence of an inaccessible cardinal it is consistent that $0<\left[2^{\omega_{1}}\right]_{L}^{\omega_{1}} \equiv\left[2^{\omega_{1}}\right]_{N}^{\omega_{1}} \equiv\left[2^{\omega_{1}}\right]_{0}^{\omega_{1}}<1$ (Theorem 4.2).

(d) Assuming the existence of a Mahlo cardinal it is consistent that $0 \leq\left[2^{\omega_{1}}\right]_{L}^{\omega_{1}} \equiv\left[2^{\omega_{1}}\right]_{N}^{\omega_{1}}<\left[2^{\omega_{1}}\right]_{0}^{\omega_{1}}<1$ (Theorem 5.14).

We do not know the answer to the following:

QUESTION 1. Does the statement $\left[2^{\omega_{1}}\right]_{L}^{\omega_{1}}<\left[2^{\omega_{1}}\right]_{0}^{\omega_{1}}$ decide whether $0<$ $\left[2^{\omega_{1}}\right]_{L}^{\omega_{1}}$ or $0 \equiv\left[2^{\omega_{1}}\right]_{L}^{\omega_{1}}$ ?

Another aspect of our results is more logical. It was Shelah [24] who suggested considering a generalized second order quantifier "for club many countable subsets $X \ldots$.."; this logic was later investigated in [2]. However when talking about topological spaces we do not have at hand any structure in the sense of universal algebra. Nevertheless one can note (Lemma 2.1) a link between a general quantifier in the sense "for club many subsets" and the general quantifier over models meaning "for any model $M \prec H(\theta)$ for $\theta$ large enough such that all relevant objects are in $M \ldots$. . In this sense the existential quantifier (i.e., the existence of a model) corresponds to the existence of stationary many subsets. Thus to prove our results about relations between the families we are considering, one needs to prove topological properties of $2^{\omega_{1}} \cap M$ for an elementary submodel $M$.

Actually the elementary submodels were the source of the research leading to this paper. In [18] the topological operation of taking a submodel of a topological space was introduced. Roughly, given a topological space $(X, \tau)$ 
and an elementary submodel $M \prec H(\theta)$ for a sufficiently large $\theta$ we can consider the space $(X \cap M, \tau \cap M)$ as a submodel of $X$. In our case, when $\omega_{1} \subseteq M$, this space is simply the set $M \cap 2^{\omega_{1}}$ with the subspace topology (since $2^{\omega_{1}}$ has a base of size $\omega_{1}$ ). So using Lemma 2.1, the results (a)-(d) above are really about the existence or nonexistence of topological submodels of the space $2^{\omega_{1}}$ with given topological properties. For instance, we will prove: it is independent of $Z F C$ whether $2^{\omega_{1}} \cap M$ is not normal for every elementary submodel $M$ such that $\omega_{1} \subseteq M$ and $|M|=\omega_{1}$ (Corollary 3.3 and Theorem 4.2).

The third aspect of our work is related to forcing, namely to the question of preservation of various properties by forcing. We consider three properties:

(a) $X$ is the set of all $\omega_{1}$-branches of a tree $T$ of height $\omega_{1}$. (Note that this is a property of the pair $(X, T)$.)

(b) A topological space $X$ has the Lindelöf property.

(c) The forcing $Q$ is c.c.c.

We say that $P$ does not add $\omega_{1}$-branches if it preserves (a), i.e., if (a) holds in the extension $V^{P}$ for every pair $(X, T)$ (in the ground model) satisfying (a) in the ground model. We say that $P$ preserves the Lindelof property (for compact spaces) if (b) holds in $V^{P}$ for every Lindelöf (compact) space in the ground model. Finally, we say that $P$ preserves c.c.c. if any c.c.c. forcing in the ground model stays c.c.c. in the extension $V^{P}$.

Preservation of properties (a) and (c) by c.c.c. forcing has been extensively investigated. For example, we have the following combinatorial characterizations: a c.c.c. forcing $P$ does not add $\omega_{1}$-branches if and only if the completion of $P$ does not contain a Suslin tree with dense levels (see [5]); and $P$ preserves c.c.c. if and only if $P \times Q$ is c.c.c. for each c.c.c. forcing $Q$. Also iterations with finite supports of forcings which preserve property (a) or property (d), preserve property (a) or (d) respectively (see [20], [5]). The situation with the preservation of (b) is different, e.g. at the moment we do not have combinatorial characterizations. For the sake of our consistency proofs, we prove results from which we can conclude the following:

(1) If a c.c.c. forcing $P$ does not preserve the Lindelöf property of compact spaces, then there is an integer $n$ such that $P^{n}$ is not c.c.c. Thus it is consistent that all c.c.c. forcings preserve the Lindelöf property of compact spaces (Theorem 4.4).

(2) Under $\mathrm{CH}$ there is a c.c.c. forcing $P$ which does not preserve the Lindelöf property of compact spaces and which does not add $\omega_{1}$-branches, and Cohen forcing preserves the property of $P$ of not adding $\omega_{1}$-branches (Corollary 5.10).

(3) There is a proper forcing which does not preserve the Lindelöf property of compact spaces and which does not add $\omega_{1}$-branches (Corollary 5.11 ). 
On the other hand it is easy to see that

(4) Every forcing which preserves $\omega_{1}$ and adds $\omega_{1}$-branches destroys the Lindelöf property of a compact space (Fact 5.12).

In the light of these results it is natural to ask the following questions which we were unable to answer:

QUESTION 2. Is there $n \in \omega$ such that if $P$ does not preserve the Lindelöf property of compact spaces, then $P^{n}$ is not c.c.c.? Is this $n=2$ ?

QUESTION 3. Characterize, in a combinatorial language, forcings which preserve the Lindelof property of compact spaces.

The notation used in this paper is standard and follows, e.g., [20] or [14].

Let us fix some more specific notation related to the partial order of the Cohen forcing $C(\alpha)$ or to the product topology on $2^{\alpha}$. For an ordinal $\gamma$, let

$$
C(\gamma)=\left\{p: p \subset f, f \in 2^{\gamma} \text { and }|p|<\omega\right\} .
$$

The elements of the forcing $C(\gamma)$ can be considered as the codes for basic clopen subsets in the product topology on $2^{\gamma}$. The coding is defined in the natural way: for $d \in C(\beta)$ and $D \subset C(\beta)$, let

$$
[d]_{\gamma}=\left\{f \in 2^{\gamma}: d \subset f\right\} \quad \text { and } \quad[D]_{\gamma}=\bigcup\left\{[d]_{\gamma}: d \in D\right\} \text {. }
$$

When $\gamma=\omega_{1}$ we will simply write $[d]$ or $[D]$. Note that these sets may be empty, for instance $[d]_{\gamma}=\emptyset$ when $d \in C(\beta)-C(\gamma)$. Considering this unnatural case of $\gamma \leq \beta$ will simplify some statements. It is clear that predense sets in $C(\gamma)$ code dense open sets in $2^{\gamma}$.

Another group of symbols is related to the internal structure of the settheoretic universes. By the axiom of regularity, the relation $\in$ is well-founded and so for each set $x$ we can define its transitive closure tc $(x)$, i.e. the set consisting of all elements of $x$ and their elements and their elements, etc. The cardinality of $\operatorname{tc}(x)$ is called the hereditary cardinality of $x$. It turns out that the class $H(\theta)$ of all sets of hereditary cardinality less than $\theta$ is a set, and a set which can be used to represent any given phenomenon happening in the set-theoretic universe provided $\theta$ is large enough (see for example [20], Theorem IV 7.5).

Finally, let us mention that intervals like $[\alpha, \beta)$ or $(\alpha, \beta)$ are considered with respect to the usual order of the ordinals.

2. Models and subfamilies of $\left[2^{\omega_{1}}\right]^{\omega_{1}}$. The link between models and families of $[X]^{\omega_{1}}$ modulo the club filter is explained by the following lemma:

Lemma 2.1. Suppose $\phi$ is a formula of ZFC. The set

$$
\left\{Y \in\left[2^{\omega_{1}}\right]^{\omega_{1}}: \phi(Y)\right\}
$$

includes a club set in $\left[2^{\omega_{1}}\right]^{\omega_{1}}$ if and only if there is $\theta$ large enough and $B \in[H(\theta)]^{\omega_{1}}$ such that whenever $M \prec H(\theta), B \subseteq M,|M|=\omega_{1}$, then 
$\phi\left(M \cap 2^{\omega_{1}}\right)$. Equivalently, the set

$$
\left\{Y \in\left[2^{\omega_{1}}\right]^{\omega_{1}}: \phi(Y)\right\}
$$

is stationary in $\left[2^{\omega_{1}}\right]^{\omega_{1}}$ if and only if for any $\theta$ and $B \in[H(\theta)]^{\omega_{1}}$ there exists $M \prec H(\theta)$ with $B \subseteq M$ and $|M|=\omega_{1}$ such that $\phi\left(M \cap 2^{\omega_{1}}\right)$.

Proof. It is clear that the two statements are equivalent. Also the reverse implication of the first equivalence follows from the fact that the collection of elementary submodels of $H(\theta)$ (including $B$ ) forms a club in $[H(\theta)]^{\omega_{1}}$ and the intersections of this club with $2^{\omega_{1}}$ forms a club in $\left[2^{\omega_{1}}\right]^{\omega_{1}}$ (see [3]).

For the forward implication of the first equivalence, let $C \subseteq\left[2^{\omega_{1}}\right]^{\omega_{1}}$ be a club as in the assumption and let $\theta$ be such that $2^{\omega_{1}}, C \in H(\theta)$. We use the idea of the proof of Kueker's result (see [3]), so let $g:\left[2^{\omega_{1}}\right]^{<\omega} \rightarrow C$ be a function such that $g(x) \supseteq x \cup \bigcup\{g(y): y \subset x\}$ and $g(\emptyset)$ is anything. Let $M \prec H(\theta)$ be such that $g, 2^{\omega_{1}}, C \in M, \omega_{1} \subseteq M$ and $|M|=\omega_{1}$. Note that for any countable $A \subseteq 2^{\omega_{1}}$ the family $C_{A}=\left\{g(x): x \in[A]^{<\omega}\right\}$ is countable, directed and included in $C$, so there is a cofinal chain in this family and consequently $\bigcup C_{A} \in C$. Let $\left(A_{\alpha}\right)_{\alpha<\omega_{1}}$ be an increasing chain of countable subsets of $M \cap 2^{\omega_{1}}$ with union $M \cap 2^{\omega_{1}}$. Then $\left(\bigcup C_{A_{\alpha}}\right)_{\alpha<\omega_{1}}$ forms an increasing chain in $C$ and so its union $C_{M}$ is in $C$. To note that $C_{M}=M \cap 2^{\omega_{1}}$ it is enough to see that $x \subseteq g(x) \subseteq M \cap 2^{\omega_{1}}$ for each $x \in\left[M \cap 2^{\omega_{1}}\right]^{<\omega}$, which follows from the fact that $g \in M$ and $\omega_{1} \subseteq M$. Thus $\phi\left(M \cap 2^{\omega_{1}}\right)$ holds, which completes the proof of the lemma.

We will now investigate normality and the Lindelöf property of certain special subspaces of $2^{\omega_{1}}$. We will actually show that these two properties are equivalent for such subspaces and we will give a characterization for it. From now on $M$ will always be a model of sufficiently large fragments of ZFC (or ZFC without the power set axiom) such that $\omega_{1} \subseteq M$ and with all relevant objects in $M$. This in particular implies that $M \cap 2^{\omega_{1}}$ is dense in $2^{\omega_{1}}$. The typical cases we are interested in are when $M$ is an elementary submodel of $H(\theta)$ for some $\theta$ and when $M$ is the ground model in a generic extension. For a set $K \subseteq 2^{\omega_{1}}$ and an ordinal $\alpha<\omega_{1}$ we let $K_{\alpha}=\{f\lceil\alpha: f \in K\}$. We can obtain the following characterization (discussions of the first author with S. Watson helped getting it):

THEOREM 2.2. $2^{\omega_{1}} \cap M$ is normal if and only if for every compact space $K \subseteq 2^{\omega_{1}}$ satisfying $K \cap M=\emptyset$ there is $\alpha<\omega_{1}$ such that $K_{\alpha} \cap M=\emptyset$.

We first show

Lemma 2.3. Suppose that $Y \subseteq 2^{\omega_{1}}$ is a dense subspace. Also suppose that there are closed disjoint subsets $F_{0}$ and $F_{1}$ of $Y$ such that for every $\alpha<\omega_{1},\left(F_{0}\right)_{\alpha} \cap\left(F_{1}\right)_{\alpha} \neq \emptyset$. Then $F_{0}$ and $F_{1}$ cannot be separated by open sets in $Y$ and therefore $Y$ is not normal. 
Proof. We have to show that $F_{0}$ and $F_{1}$ cannot be separated in $Y$. Note that, since $Y$ is dense in $2^{\omega_{1}}$, it is enough to show that we cannot separate $F_{0}$ and $F_{1}$ by open sets in $2^{\omega_{1}}$. Suppose that there are open disjoint subsets $U$ and $V$ of $2^{\omega_{1}}$ such that $F_{0} \subseteq U$ and $F_{1} \subseteq V$. By our assumptions on $F_{0}$ and $F_{1}$, for each $\alpha<\omega_{1}$, we can fix $g_{\alpha} \in\left(F_{0}\right)_{\alpha} \cap\left(F_{1}\right)_{\alpha}$. Let $h_{\alpha} \in F_{0}$ be such that $h_{\alpha}\left\lceil\alpha=g_{\alpha}\right.$. For each $\alpha<\omega_{1}$, fix a basic open set $\left[p_{\alpha}\right]=\left\{f \in 2^{\omega_{1}}: p_{\alpha} \subseteq f\right\}$ such that $h_{\alpha} \in\left[p_{\alpha}\right] \subseteq U$. Note that $\left[p_{\alpha}\right] \subseteq U$ implies $\left[p_{\alpha}\right] \cap F_{1}=\emptyset$.

Claim. We need uncountably many $\left[p_{\alpha}\right]$ 's to cover $\left\{h_{\alpha}: \alpha<\omega_{1}\right\}$.

Proof. Fix $\left\{\left[p_{\alpha_{n}}\right]: n \in \omega\right\}$ and take $\delta>\sup \bigcup\left\{\operatorname{dom}\left(p_{\alpha_{n}}\right): n \in \omega\right\}$. We will show that $h_{\delta} \notin\left[p_{\alpha_{n}}\right]$ for every $n \in \omega$. Suppose $h_{\delta} \in\left[p_{\alpha_{n}}\right]$. Then $p_{\alpha_{n}}=$ $h_{\delta}\left\lceil\operatorname{dom}\left(p_{\alpha_{n}}\right)\right.$. Since $\delta>\max \operatorname{dom}\left(p_{\alpha_{n}}\right)$, we have $p_{\alpha_{n}}=g_{\delta}\left\lceil\operatorname{dom}\left(p_{\alpha_{n}}\right)\right.$. But now take $f_{\delta} \in F_{1}$ such that $f_{\delta}\left\lceil\delta=g_{\delta}\right.$. Then $f_{\delta} \in\left[p_{\alpha_{n}}\right] \cap F_{1}$, a contradiction.

We then fix $\left\{p_{\alpha}: \alpha<\omega_{1}\right\}$ such that $\left\{\left[p_{\alpha}\right]: \alpha<\omega_{1}\right\}$ covers $\left\{h_{\alpha}: \alpha<\omega_{1}\right\}$. Without loss of generality, we can suppose that $\left\{d_{\alpha}: \alpha<\omega_{1}\right\}$, where $d_{\alpha}=$ $\operatorname{dom}\left(p_{\alpha}\right)$ for each $\alpha<\omega_{1}$, forms a $\Delta$-system with root $d$. Since $d$ is finite, $2^{|d|}$ is finite. So we can also suppose that $p_{\alpha}\left\lceil d=p\right.$ for every $\alpha<\omega_{1}$, for some fixed $p$.

Pick $\delta>\max d$. Note that $g_{\delta}\left\lceil d=p_{\delta}\left\lceil d=p\right.\right.$. If $f_{\delta} \in F_{1}$ is such that $f_{\delta}\left\lceil\delta=g_{\delta}\right.$, then there is a basic open set $[q] \subseteq V$ such that $f_{\delta} \in[q]$. But then $[q] \cap\left[p_{\alpha}\right]=\emptyset$ for every $\alpha<\omega_{1}$, which implies that $\operatorname{dom}(q) \cap \operatorname{dom}\left(p_{\alpha}\right) \neq \emptyset$ for every $\alpha<\omega_{1}$.

Now, if $\beta>\delta$, then $p_{\beta}\left\lceil d=p_{\delta}\left\lceil d=g_{\delta}\lceil d=q\lceil\right.\right.$. We must then have $\left(\operatorname{dom}(q) \cap \operatorname{dom}\left(p_{\beta}\right)\right) \backslash d \neq \emptyset$ for every $\beta>\delta$, a contradiction.

Proof of the Theorem. Here we will only prove one direction. For the other direction, we can actually get a stronger result, which will be given in the next theorem. Suppose there is a compact $K$ such that $K \cap M=\emptyset$ but $K_{\alpha} \cap M \neq \emptyset$ for every $\alpha<\omega_{1}$. By the previous lemma we just have to define the two closed sets satisfying its hypothesis. For $i=0,1$ define $F_{i}$ to be the set

$$
\left\{f \in 2^{\omega_{1}} \cap M: \exists \alpha<\omega_{1} \exists g \in K_{\alpha} \cap M \quad f\lceil\alpha=g\lceil\alpha \& \forall \beta>\alpha f(\beta)=i\} .\right.
$$

We first show that $\bar{F}_{0} \cap \bar{F}_{1}=\emptyset$, with the closures taken in $2^{\omega_{1}} \cap M$. For that we fix $f \in M$. Then, by our assumption on $K, f \notin K$. Therefore there is a basic open set $[p]$ such that $f \in[p]$ and $[p] \cap K=\emptyset$, where $p$ is a finite function from $\omega_{1}$ to 2 . Pick $\alpha>\max \operatorname{dom}(p)$ and define $q=p \cup\{(\alpha, f(\alpha))\}$.

Claim. $f(\alpha)=1-i$ implies that $[q] \cap F_{i}=\emptyset$.

Proof. Fix $h \in F_{i}$. By the definition of $F_{i}$, there are $\beta<\omega_{1}$ and $g \in$ $K_{\beta} \cap M$ such that $h \uparrow \beta=g\lceil\beta$ and $h(\delta)=i$ for every $\delta>\beta$. We have two possibilities: 
CASE 1: $\beta \geq \alpha$. Then, since $g \in K_{\beta} \cap M,[p] \cap K=\emptyset$ and $\beta>$ $\max \operatorname{dom}(p)$, we have $h \notin[p]$. Therefore $h \notin[q]$.

CASE 2: $\beta<\alpha$. Then, since $h(\delta)=i$ for every $\delta>\beta$ and $\beta<\alpha$, we have $h(\alpha)=i$ and therefore $h \notin[q]$.

Thus for every $f \in M$ there is $\alpha<\omega_{1}$ such that $f(\alpha)=1-i$ implies $f \notin \bar{F}_{i}$, which implies that $\bar{F}_{0} \cap \bar{F}_{1}=\emptyset$.

We now look at the Lindelöf property. In particular, the next theorem finishes the proof of the previous theorem.

THEOREM 2.4. Let $Y$ be any subspace of $2^{\omega_{1}}$. Then $Y$ is Lindelöf if and only if for every compact $K \subset 2^{\omega_{1}}$ with $K \cap Y=\emptyset$ there is $\alpha<\omega_{1}$ such that $K_{\alpha} \cap Y_{\alpha}=\emptyset$.

Proof. For one direction, we want to show that $Y$ is Lindelöf. Let $\mathcal{C}$ be an open cover of $Y$. If $\bigcup \mathcal{C}=2^{\omega_{1}}$, it has a finite subcover (since $2^{\omega_{1}}$ is compact) and we are done. If not, let $K=2^{\omega_{1}} \backslash \bigcup \mathcal{C}$. Then $K$ is a compact set disjoint from $Y$, so fix $\alpha<\omega_{1}$ such that $K_{\alpha} \cap Y_{\alpha}=\emptyset$.

Since $K_{\alpha}$ is a closed subset of $2^{\alpha}$ and $\alpha$ is countable, $K_{\alpha}$ is a $G_{\delta}$-set in $2^{\alpha}$. So fix open sets (in $\left.2^{\alpha}\right) W_{n}$, for $n \in \omega$, such that $K_{\alpha}=\bigcap_{n \in \omega} W_{n}$. Let $V_{n}$ be the open set of $2^{\omega_{1}}$ which is the inverse image of $W_{n}$ by the projection map onto $2^{\alpha}$, i.e., $V_{n}=\operatorname{proj}_{\alpha}^{-1}\left(W_{n}\right)$.

Let $F=\bigcup_{n \in \omega}\left(2^{\omega_{1}} \backslash V_{n}\right)$. Note that $F$ is an $F_{\sigma}$ in $2^{\omega_{1}}$, and therefore it has the Lindelöf property. Also, since $K_{\alpha} \cap Y_{\alpha}=\emptyset$, we see that $Y \subseteq F$. Finally, by the definition of $F$ and the $V_{n}$ 's, we have $F \subseteq \cup \mathcal{C}$. These three facts imply that there is $\mathcal{C}^{\prime} \subseteq \mathcal{C}$ countable such that $Y \subseteq \cup \mathcal{C}^{\prime}$, and therefore $Y$ is Lindelöf.

For the other implication, suppose that a compact set $K \subset 2^{\omega_{1}}$ is such that $K \cap Y=\emptyset$ but $K_{\alpha} \cap Y_{\alpha} \neq \emptyset$ for every $\alpha<\omega_{1}$. Then for each $\alpha<\omega_{1}$, we can fix $f_{\alpha} \in 2^{\omega_{1}}$ such that $f_{\alpha}\left\lceil\alpha \in K_{\alpha} \cap Y_{\alpha}\right.$. Clearly, these functions can be assumed to be distinct. Let $g$ be any complete accumulation point of $\left\{f_{\alpha}: \alpha<\omega_{1}\right\}$.

We will show that $g$ is a complete accumulation point of $K$. Then since $K$ is closed we will have $g \in K$. But this implies that $g \notin Y$ and therefore $\left\{f_{\alpha}: \alpha<\omega_{1}\right\}$ does not have any complete accumulation point in $Y$, which completes the proof.

To show that $g$ is a complete accumulation point of $K$, fix $p \subset g$ finite and let $[p]$ be a basic open set. Since $g$ is a complete accumulation point of $\left\{f_{\alpha}: \alpha<\omega_{1}\right\}$, we have $\left|[p] \cap\left\{f_{\alpha}: \alpha<\omega_{1}\right\}\right|=\omega_{1}$. Pick $\beta>\max \operatorname{dom}(p)$. Then $f_{\beta} \in[p]$ implies that $f_{\beta}\left\lceil\beta \supseteq g\left\lceil\operatorname{dom}(p)\right.\right.$. But there is $g_{\beta} \in K$ such that $g_{\beta}\left\lceil\beta=f_{\beta}\left\lceil\beta\right.\right.$. Thus, since $\beta>\max \operatorname{dom}(p)$, we have $g_{\beta} \in[p]$. Since this is true for every $\beta$ such that $f_{\beta} \in[p]$, we conclude that $|[p] \cap K|=\omega_{1}$. 
Corollary 2.5. For $M$ as before, $2^{\omega_{1}} \cap M$ is Lindelöf if and only if it is normal. In particular:

(a) if $M$ is an elementary submodel of $H(\theta)$ for some $\theta \geq\left(2^{\omega_{1}}\right)^{+}$such that $\omega_{1} \subset M$, then the space $2^{\omega_{1}} \cap M$ is Lindelof if and only if it is normal;

(b) if $G$ is a P-generic for a partial order $P$ which preserves $\omega_{1}$, then in $V^{P}$ the space $2^{\omega_{1}} \cap V$ is Lindelöf if and only if it is normal.

COROLlaRY 2.6. $\left[2^{\omega_{1}}\right]_{L}^{\omega_{1}}$ and $\left[2^{\omega_{1}}\right]_{N}^{\omega_{1}}$ are club equivalent, i.e., $\left[2^{\omega_{1}}\right]_{L}^{\omega_{1}} \equiv$ $\left[2^{\omega_{1}}\right]_{N}^{\omega_{1}}$.

The proof of the next theorem is in [17], or in a more general form, in [18]:

Proposition 2.7. For a cardinal $\theta \geq\left(2^{\omega_{1}}\right)^{+}$, there is a model $M \prec$ $H(\theta)$ such that $|M|=\omega_{1}$ and $2^{\omega_{1}} \cap M$ is not closed under $\omega_{1}$-limits.

Corollary 2.8. $\left[2^{\omega_{1}}\right]_{0}^{\omega_{1}}$ and so $\left[2^{\omega_{1}}\right]_{L}^{\omega_{1}},\left[2^{\omega_{1}}\right]_{N}^{\omega_{1}}$ are co-stationary.

Corollary 2.9. $0 \leq\left[2^{\omega_{1}}\right]_{L}^{\omega_{1}} \equiv\left[2^{\omega_{1}}\right]_{N}^{\omega_{1}} \leq\left[2^{\omega_{1}}\right]_{0}^{\omega_{1}}<1$.

3. Subspaces of $2^{\omega_{1}}$ and Kurepa trees. Note that in Theorem 2.2, to deduce that $2^{\omega_{1}} \cap M$ is not normal, the compact set $K$ could in particular be a single point. Actually, a similar proof (but simpler) yields the following result:

THEOREM 3.1. Let $M$ be as in the previous section. If $\kappa \subseteq M$ and there is $f \in 2^{\kappa}$ such that for some ordinal $\alpha$ of uncountable cofinality we have $f\left\lceil\beta \in M\right.$ for every $\beta<\alpha$ and $f\left\lceil\alpha \notin M\right.$, then $2^{\kappa} \cap M$ is not normal.

It is natural to ask when there is such a function. For the case $\kappa=\omega_{1}$ we have the following characterization:

THEOREM 3.2. The statement "for every elementary submodel $M$ of $H(\theta)$ for some $\theta \geq\left(2^{\omega_{1}}\right)^{+}$with $\omega_{1} \subseteq M,|M|=\omega_{1}$, there is $f \notin M$ such that $f\left\lceil\alpha \in M\right.$ for every $\alpha<\omega_{1}$ " is true if and only if there is a weak Kurepa tree, i.e., a tree of height $\omega_{1}$ with levels of cardinality $\leq \omega_{1}$ and with more than $\omega_{1}$ uncountable branches.

Proof. First, suppose there are no weak Kurepa trees. Then, in particular, there are no weak Kurepa trees $T \subseteq 2^{\omega_{1}}$. We will show that there is an elementary submodel $M$ of $H(\theta)$ with $\omega_{1} \subseteq M,|M|=\omega_{1}$ such that for every $f \notin M$ there is $\alpha<\omega_{1}$ such that $f\lceil\alpha \notin M$. We will identify a cofinal branch $b$ through the tree $2^{<\omega_{1}}$ with the corresponding function $f_{b} \in 2^{\omega_{1}}$. We can construct a chain $\left\{M_{n}: n \in \omega\right\}$ of elementary submodels such that for every $n \in \omega, M_{n}$ is an elementary submodel of $M_{n+1},\left|M_{n}\right|=\omega_{1}, \omega_{1} \subseteq M_{n}$ and

$$
\left\{f \in 2^{\omega_{1}}: f\left\lceil\alpha \in M \text { for every } \alpha \in \omega_{1}\right\} \subseteq M_{n+1}\right. \text {. }
$$


Note that the construction is possible since there are no weak Kurepa trees, and therefore $2^{<\omega_{1}} \cap M$ has at most $\omega_{1}$ cofinal branches. We claim that $M=\bigcup_{n \in \omega} M_{n}$ is as we want. Suppose $f \in 2^{\omega_{1}} \backslash M$. Then for each $n \in \omega$ we can take $\alpha_{n} \in \omega_{1}$ minimum such that $f\left\lceil\alpha_{n} \notin M_{n}\right.$ (such an $\alpha_{n}$ exists because otherwise we would have $\left.f \in M_{n+1} \subseteq M\right)$. Take $\alpha=\sup \left\{\alpha_{n}: n \in \omega\right\}$. Since $f\left\lceil\alpha \in M_{n}\right.$ implies $f\left\lceil\alpha_{n} \in M_{n}\right.$, we see that $f\left\lceil\alpha \notin M_{n}\right.$ for every $n \in \omega$. Thus $f\lceil\alpha \notin M$.

Suppose now that there is a weak Kurepa tree. Then there is one which is a subtree of $2^{<\omega_{1}}$. Let $M \prec H(\theta)$ be such that $\omega_{1} \subseteq M$ and $|M|=\omega_{1}$. By elementarity, there is $T \in M$ such that $T \subseteq 2^{<\omega_{1}}$ and $T$ is a weak Kurepa tree. Note that $|T|=\omega_{1}$ and therefore $T \subseteq M$ (since $\omega_{1} \subseteq M$ ). Thus $2^{<\omega_{1}} \cap M$ contains a weak Kurepa tree, so it has $>\omega_{1}$ branches. Since $|M|=\omega_{1}$, this implies that there must be a branch $b$ of $2^{<\omega_{1}} \cap M$ which is not in $M$. Then the corresponding function $f_{b} \notin M$, but $f_{b}\lceil\alpha \in M$ for every $\alpha \in \omega_{1}$.

Corollary 3.3. Suppose that $w K H$ holds. If $M$ is an elementary submodel of $H(\theta)$ for some $\theta \geq\left(2^{\omega_{1}}\right)^{+}$of size $\omega_{1}$ and $\omega_{1} \subseteq M$, then $2^{\omega_{1}} \cap M$ is not closed under limits of $\omega_{1}$-sequences and therefore it is nonnormal and non-Lindelöf.

Corollary 3.4. wKH implies that $0 \equiv\left[2^{\omega_{1}}\right]_{L}^{\omega_{1}} \equiv\left[2^{\omega_{1}}\right]_{N}^{\omega_{1}} \equiv\left[2^{\omega_{1}}\right]_{\circ}^{\omega_{1}}<1$.

Corollary 3.5. ᄀwKH implies that $\left[2^{\omega_{1}}\right]_{0}^{\omega_{1}}$ is stationary, i.e., that $0<$ $\left[2^{\omega_{1}}\right]_{0}^{\omega_{1}}<1$

4. Forcing results by preservation of the Lindelöf property. For the first result of this section we will use the Mitchell model and a lemma due to A. Dow. The Mitchell forcing $M i_{\kappa}$ (see [22] for details) is an iteration with mixed supports of forcings that add Cohen reals and forcings which collapse $2^{\omega}$ to $\omega_{1}$. The iteration $\left(M i_{\alpha}, \dot{M} i^{\alpha}\right)_{\alpha<\kappa}$ is of length $\kappa$, where $\kappa$ is an inaccessible cardinal. The Cohen part is taken with finite supports and the collapse part is taken with countable supports. It is true (see [22] or [8]) that in $V^{M i_{\alpha}}$ for $\alpha<\kappa$ the remaining part of the forcing looks like the entire Mitchell forcing. For the convenience of the reader we list after [8] the properties of Mitchell forcing:

(i) if $G$ is $M i_{\kappa}$-generic, for $\kappa$ strongly inaccessible, then $V[G]$ satisfies $\omega_{1}=\omega_{1}^{V}, 2^{\omega}=\omega_{2}=\kappa$;

(ii) if $\lambda<\kappa$ is even, then $M i_{\kappa}$ is a forcing isomorphic to $M i_{\lambda} * M i_{\kappa^{\prime}}$ where $\lambda+\kappa^{\prime}=\kappa$;

(iii) there is a $M i_{\kappa}$-name, $\dot{R}$, of a poset such that $M i_{\kappa} * \dot{R}$ is a forcing isomorphic to $C(\mu) \times Q$ for some $\omega_{1}$-closed poset $Q$ and some uncountable $\mu$; 
(iv) there is a $M i_{\kappa}$-name of a poset $\dot{R}$ and a $C\left(\omega_{1}\right)$-name of a poset $\dot{Q}$ such that $M i_{\kappa} * \dot{R}$ is a forcing isomorphic to $C\left(\omega_{1}\right) *\left(\dot{Q} \times C\left(\kappa-\omega_{1}\right)\right)$ such that $C\left(\omega_{1}\right) \Vdash \dot{Q}$ is $\omega_{1}$-closed.

We will also need (see [22], Lemma 3.3)

(v) if $\kappa$ is an inaccessible cardinal, then $M i_{\kappa}$ satisfies the $\kappa$-c.c.

Alan Dow [8] proved that forcing with Mitchell forcing does not destroy the Lindelöf property, so $2^{\omega_{1}} \cap V^{M i_{\alpha}}$, which is a compact space in $V^{M i_{\alpha}}$, remains Lindelöf in $V^{M i_{\kappa}}$. On the other hand for $\alpha<\kappa,\left|V^{M i_{\alpha}} \cap 2^{\omega_{1}}\right|<\kappa$ by the inaccessibility of $\kappa$, so by (i) this set is in $\left[2^{\omega_{1}}\right]^{\omega_{1}}$ (in $V^{M i_{\kappa}}$ ). This is sufficient for describing the relationships that hold in the Mitchell model among the families we are considering. Let $\kappa$ be an inaccessible cardinal. We define by induction, in $V$, a sequence $\left(\dot{X}_{\alpha}: \alpha<\kappa\right)$ of $M i_{\kappa}$-names for subsets of $2^{\omega_{1}}$ of cardinality $\omega_{1}$ and a sequence $\left(\gamma_{\alpha}: \alpha<\kappa\right)$ of ordinals in the following way: $M i_{\kappa} \Vdash \dot{X}_{\alpha}=\bigcup_{\beta<\alpha} \dot{X}_{\beta}$ and $\gamma_{\alpha}=\sup \left\{\gamma_{\beta}: \beta<\alpha\right\}$ for $\alpha$ limit and if $\alpha=\beta+1$ for some $\beta$ we require $M i_{\kappa} \Vdash \dot{X}_{\alpha}=2^{\omega_{1}} \cap V^{M i_{\gamma_{\alpha}}}$ for $\gamma_{\alpha}$ minimal greater than $\gamma_{\beta}$ such that $M i_{\kappa} \Vdash \dot{X}_{\beta} \subset V^{M i_{\gamma_{\alpha}}}$. Note that this $\gamma_{\alpha}$ is well defined by (v) as $X_{\beta}$ is of cardinality $\omega_{1}$ in $V^{M i_{\kappa}}$ (by (i)).

LEMMA 4.1. In $V^{M i_{\kappa}}$, if $\operatorname{cf}(\alpha) \leq \omega$ or $X_{\alpha}=2^{\omega_{1}} \cap V^{M i_{\gamma_{\alpha}}}$, then $X_{\alpha} \in$ $\left[2^{\omega_{1}}\right]_{L}^{\omega_{1}}$. If $\operatorname{cf}(\alpha)>\omega$ and $X_{\alpha} \neq 2^{\omega_{1}} \cap V^{M i_{\gamma_{\alpha}}}$, then $X_{\alpha} \notin\left[2^{\omega_{1}}\right]_{0}^{\omega_{1}}$.

Proof. If $\alpha$ is a successor or $X_{\alpha}=2^{\omega_{1}} \cap V^{M i_{\gamma_{\alpha}}}$, then $X_{\alpha}=2^{\omega_{1}} \cap V^{M i_{\gamma_{\alpha}}}$ and as $2^{\omega_{1}} \cap V^{M i_{\gamma_{\alpha}}}$ is a compact space in $V^{M i_{\gamma_{\alpha}}}$, by Dow's result we deduce that $X_{\alpha}$ is Lindelöf in $V^{M i_{\kappa}}$.

If $\alpha$ is of countable cofinality, then $X_{\alpha}$ is the union of a countable family of Lindelöf subspaces of the form $X_{\beta+1}=2^{\omega_{1}} \cap V^{M i_{\gamma_{\beta+1}}}$ for $\beta<\alpha$, so it is Lindelöf as well.

If $\operatorname{cf}(\alpha)>\omega_{1}$ and $X_{\alpha} \neq 2^{\omega_{1}} \cap V^{M i_{\gamma_{\alpha}}}$, then pick $x \in 2^{\omega_{1}} \cap\left(V^{M i_{\gamma_{\alpha}}} \backslash\right.$ $\left.\bigcup_{\beta<\alpha} V^{M i_{\gamma_{\beta}}}\right)$. As conditions of $M i_{\kappa}$ have at most countable supports, each condition of the generic set deciding the $x(\xi)$ for $x \in 2^{\omega_{1}} \cap V^{M i_{\gamma_{\alpha}}}$ and $\xi \in \omega_{1}$ is in $M i_{\delta}$ for some $\delta<\gamma_{\alpha}$. As $\operatorname{cf}(\alpha)>\omega$, it stays such in the generic extension (as $\omega_{1}$ is preserved), so any countably many of these conditions are in some $M i_{\delta}$ for $\delta<\gamma_{\alpha}$ and so $x\left\lceil\xi^{\frown} 0 \in X_{\alpha}\right.$ for every countable $\xi$. This implies that $X_{\alpha} \notin\left[2^{\omega_{1}}\right]_{0}^{\omega_{1}}$ as required.

TheOREM 4.2. Assuming the existence of an inaccessible cardinal it is consistent that

$$
0<\left[2^{\omega_{1}}\right]_{L}^{\omega_{1}} \equiv\left[2^{\omega_{1}}\right]_{N}^{\omega_{1}} \equiv\left[2^{\omega_{1}}\right]_{\circ}^{\omega_{1}}<1 .
$$

Proof. Let us work in the Mitchell model and use the previous lemma. Clearly the $X_{\alpha}$ 's form a club subset of $\left[2^{\omega_{1}}\right]^{\omega_{1}}$. The previous lemma and Corollary 2.9 imply all the required relations. 
Corollary 4.3. Assuming the existence of an inaccessible cardinal it is consistent that there is an elementary submodel $M$ of size $\omega_{1}$ such that $\omega_{1} \subseteq M$ and $2^{\omega_{1}} \cap M$ is Lindelöf and therefore normal.

Using the same preservation argument of A. Dow [8], it was noted by Baumgartner and Tall [4] (using weakly compact cardinals) that it is consistent that Lindelöf spaces of character $\leq \omega_{1}$ and weight $\leq \omega_{2}$ have Lindelöf subspaces of size $\omega_{1}$. Restricting the attention to compact spaces of weight $\omega_{1}$, one can get a consistency reflection result just from the existence of an inaccessible. Indeed, the proof of the previous lemma works in the case of any compact space of weight $\omega_{1}$, as we can code it with a family of clopen subsets of $[0,1]^{\omega_{1}}$ of cardinality $\omega_{1}$, i.e., in the Mitchell model, we can obtain $0<[X]_{L}^{\omega_{1}}$ for any compact space of weight $\omega_{1}$ and cardinality greater than $\omega_{1}$.

One can eliminate the large cardinals completely at the price of the assumption of first countability of the space. By the Arkhangel'skil theorem, it makes sense to talk about $[X]_{L}^{\omega_{1}}$, for $X$ first countable, only in the presence of the negation of $\mathrm{CH}$. Various models of $\neg \mathrm{CH}$ where all first countable compact spaces $X$ of weight $\omega_{1}$ have $[X]_{L}^{\omega_{1}}$ stationary can be provided, the easiest being the Cohen model (using the fact that the Cohen forcing preserves the Lindelöf property; see [8], Lemma 3.4). To build other models one needs stronger preservation lemmas, e.g. random reals also preserve the Lindelöf property (see [10]). But stronger (than Dow's lemma) preservation results can be proved for the Lindelöf property and compact spaces in general. Here is our strongest preservation result of this form which answers a question of Juhász (whether there is in ZFC a compact space and a c.c.c. forcing which destroys the Lindelöf property of the space [15]).

Theorem 4.4. Suppose that $X$ is a compact space and $P$ is a forcing notion such that $P$ forces that $X$ is not Lindelöf. Then there is $n<\omega$ such that $P^{n}$ does not satisfy the c.c.c. In particular $M A+\neg C H$ implies that there are no such $X$ and $P$.

Proof. Let $\mathcal{B}$ be a basis for the topology of $X$. Without loss of generality, we can assume that there is an uncountable cardinal $\kappa$ and a sequence of $P$-names $\left\{\dot{U}_{\xi}: \xi<\kappa\right\}$ for elements of $\mathcal{B}$ such that $P$ forces that $\check{X} \subseteq \bigcup\left\{\dot{U}_{\xi}\right.$ : $\xi<\kappa\}$ and that there is no countable subcover of this cover.

For $p \in P$ define

$$
V_{p}=\bigcup\left\{U: \exists \xi<\kappa p \Vdash \check{U}=\dot{U}_{\xi}\right\} .
$$

Note that, since for every $p \in P$ and $x \in X$ there are $\xi<\kappa, U \in \mathcal{B}$ and $q \leq p$ such that

$$
q \Vdash x \in \check{U}=\dot{U}_{\xi},
$$


we have

$$
X \subseteq \bigcup\left\{V_{q}: q \leq p\right\}
$$

for any $p \in P$. Using the fact that $X$ is compact we can find a finite subcover of the cover $\left\{V_{q}: q \leq p\right\}$ and using the compactness of $X$ once again for each $p \in P$ we can obtain $n(p) \in \omega, q_{1}(p), \ldots, q_{n(p)}(p) \leq p, U_{1}^{p}, \ldots, U_{n(p)}^{p} \subseteq \mathcal{B}$ and $\xi_{1}(p), \ldots, \xi_{n(p)}(p)<\kappa$ such that

$$
X \subseteq U_{1}^{p} \cup \ldots \cup U_{n(p)}^{p}
$$

and

$$
q_{i}(p) \Vdash \check{U}_{i}^{p}=\dot{U}_{\xi_{i}(p)}
$$

for all $i \leq n(p)$.

Now by induction on $\alpha<\omega_{1}$ construct a sequence $\left(p_{\alpha}: \alpha<\omega_{1}\right)$ of conditions of $P$ and a sequence $\left(x_{\alpha}: \alpha<\omega_{1}\right)$ of points of $X$ such that

$$
p_{\alpha} \Vdash \check{x}_{\alpha} \notin \bigcup\left\{\dot{U}_{\xi_{i}\left(p_{\beta}\right)}: i \leq n\left(p_{\beta}\right), \beta<\alpha\right\} .
$$

Given $\left(p_{\beta}: \beta<\alpha\right)$ and $\left(x_{\beta}: \beta<\alpha\right)$, we can easily find the next $p_{\alpha}$ and $x_{\alpha}$ as $P$ forces that $\left\{\dot{U}_{\xi}: \xi<\kappa\right\}$ has no countable subcover, so in particular $\left\{\dot{U}_{\xi_{i}\left(p_{\beta}\right)}: i \leq n\left(p_{\beta}\right), \beta<\alpha\right\}$ is not a cover of $X$.

Clearly without loss of generality we can assume that $n\left(p_{\alpha}\right)=n$ for all $\alpha<\omega_{1}$ and some $n \in \omega$. We now consider the sequence $\left\{\left(q_{1}\left(p_{\alpha}\right), \ldots, q_{n}\left(p_{\alpha}\right)\right)\right.$ : $\left.\alpha<\omega_{1}\right\} \subseteq P^{n}$ and observe that it is an uncountable antichain of $P^{n}$, which will complete the proof. Let $\beta<\alpha<\omega_{1}$. Let $i \leq n$ be such that $x_{\alpha} \in U_{i}^{p_{\beta}}$ (by $(1))$. By (2) we have

$$
q_{i}\left(p_{\beta}\right) \Vdash \check{U}_{i}^{p_{\beta}}=\dot{U}_{\xi_{i}\left(p_{\beta}\right)} .
$$

On the other hand by (3),

$$
q_{i}\left(p_{\alpha}\right) \leq p_{\alpha} \Vdash x_{\alpha} \notin \dot{U}_{\xi_{i}\left(p_{\beta}\right)}
$$

and so this implies that $q_{i}\left(p_{\beta}\right)$ and $q_{i}\left(p_{\alpha}\right)$ are incompatible.

We can use this theorem for the following simple reflection result (without large cardinals). Recall that MAK is Martin's axiom for partial orders which have property $K$.

THEOREM 4.5. $M A K+\neg C H$ is consistent with " $[X]_{L}^{\omega_{1}}$ is stationary for any compact first countable space of weight $<2^{\omega} "$.

Proof. Iterate with finite supports all c.c.c. forcings which are powerfully c.c.c., i.e., whose finite powers satisfy the c.c.c. (in particular they have property $K$ ). This preserves the Lindelöf property of compact spaces (by the previous theorem). Make the iteration of length $\omega_{2}$ consisting of forcings of size $\omega_{1}$ and start with a model of $\mathrm{CH}$. Let $X$ be a compact first countable space of weight $\omega_{1}$. Without loss of generality we can assume that $X=$ 
$[0,1]^{\omega_{1}}-\bigcup \mathcal{U}$ where $\mathcal{U}$ is a family of basic open subsets of $[0,1]^{\omega_{1}}$ determined by finitely many coordinates and open intervals with rational end-points. A code for $\mathcal{U}$ is in $V^{P_{\alpha}}$ for every $\alpha<\omega_{2}$ greater than some $\beta<\omega_{2}$ and $X_{\alpha}=\left([0,1]^{\omega_{1}}-\bigcup \mathcal{U}\right) \cap V^{P_{\alpha}}$ is a compact space in $V^{P_{\alpha}}$.

Claim. In $V^{P_{\alpha}}$ the space $X_{\alpha}$ is first countable.

Proof. Let $x \in X_{\alpha}$. Working in $V^{P}$ and using the first countability of $X$, we can find $\beta<\omega_{1}$ such that the family $\mathcal{B}_{x}^{\beta}$ of all basic open sets of $[0,1]^{\omega_{1}}$ containing $x$ determined by finitely many intervals with rational end-points rises (by intersecting its elements with $X_{\alpha}$ ) to a local basis of $x$ in $X_{\alpha}$. But this family is definable from $x$, and thus belongs to $V^{P_{\alpha}}$.

Now by Arkhangel'ski1's theorem, the cardinality of $X_{\alpha}$ is at most continuum in $V^{P_{\alpha}}$, which is $\omega_{1}$. By the previous theorem, $X_{\alpha}$ has the Lindelöf property as iterations of c.c.c. forcings whose powers are c.c.c. are powerfully c.c.c. as well. Now the $X_{\alpha}$ 's and their countable unions are in $[X]_{L}^{\omega_{1}}$ and so $[X]_{L}^{\omega_{1}}$ is stationary, as required.

Here we do not know how to add full MA without destroying the Lindelöf property of compact spaces. For example, one could do it if the answer to the following question were positive.

QUeSTION 4. Suppose P is c.c.c. and destroys the Lindelöf property of a compact space (in particular $2^{\omega_{1}}$ ). Is there a forcing $Q$ which does not destroy the Lindelof property of compact spaces (in particular $2^{\omega_{1}}$ ) such that $Q$ destroys the c.c.c. of $P$ ?

The possibility of adding MA by a c.c.c. forcing without destroying the Lindelöf property of compact spaces would mean by Theorem 4.4 that one can make (using a c.c.c. forcing) the Lindelöf property of $2^{\omega_{1}}$ indestructible by c.c.c. forcings. This could facilitate the answer to Question 1. Another natural question related to Theorem 4.5 is:

QUESTION 5. Is it consistent that $[X]_{L}^{\omega_{1}}$ is nonstationary for some first countable compact space $X$ of cardinality larger than $\omega_{1}$ ?

5. Forcing results by destroying the Lindelöf property. In this section instead of preservation results we will be more interested in destroying the Lindelöf property. This will be combined with a technology essentially due to Devlin [5] and designed in our particular case by Todorčević [29] to obtain the consistency of MA $+\neg$ wKH. First, using CH we will construct a c.c.c. forcing which destroys the Lindelöf property of $2^{\omega_{1}}$. Moreover, we need this forcing to satisfy extra properties like, for example, not adding uncountable branches. The idea relies on a phenomenon discovered by Luzin [21], fully combinatorially exploited by the Hungarian school (e.g. [11] or [9]), and later in the forcing context by Shelah in e.g. [25] and Todorčević 
in e.g. [30]. First we will need some preparatory definition and facts (about the notation, see the end of the introduction).

Definition 5.1. $D \subseteq C(\alpha)$ is called powerfully predense in $C(\alpha)$ if for all $n<\omega$ the collection $\Delta_{n}(D)=\left\{\langle d, \ldots, d\rangle \in C(\alpha)^{n}: d \in D\right\}$ is predense in $C(\alpha)^{n}$.

FACT 5.2. Suppose that $\alpha<\beta$ are ordinals. If $D$ is powerfully predense in $C(\alpha)$, then $D$ is powerfully predense in $C(\beta)$.

FACT 5.3. Suppose that $D$ is powerfully predense in $C\left(\omega_{1}\right)$. Suppose that $N \prec H(\theta)$ for $\theta \geq\left(2^{\omega_{1}}\right)^{+}$is such that $|N|=\omega_{1}$ and $D \in N$. Then there is $D^{\prime} \in[D]^{\omega}$ such that $D^{\prime}$ is powerfully predense in $C\left(\omega_{1}\right)$ and $D^{\prime} \in N \cap H\left(\omega_{1}\right)$.

Proof. By the c.c.c. of $C\left(\omega_{1}\right)^{n}$, there are countable $D_{n} \subseteq D$ such that $\Delta_{n}\left(D_{n}\right)$ are predense in $C\left(\omega_{1}\right)^{n}$. Take a countable $D^{\prime} \subseteq C\left(\omega_{1}\right)$ which includes all of them. Then $D^{\prime}$ is as required.

Our forcing will depend on two sequences, $\mathcal{M}=\left(M_{\alpha}\right)_{\alpha<\omega_{1}}$ and $\mathcal{L}=$ $\left(l_{\alpha}\right)_{\alpha<\omega_{1}}$, where $M_{\alpha}$ is a countable set such that $M_{\alpha} \cap \omega_{1}=\gamma_{\alpha} \in \omega_{1}, \gamma_{\alpha}<$ $\gamma_{\alpha+1}$ and $l_{\alpha} \in 2^{\gamma_{\alpha}}$ for all $\alpha<\omega_{1}$. Usually we will require more properties but for the definition of the forcing and the following lemma we just need these weak assumptions.

We define the forcing $P=P_{\mathcal{M}, \mathcal{L}}$, where $\mathcal{M}$ and $\mathcal{L}$ are as above, as follows: the conditions $p \in P$ are of the form $\left(a_{p}, U_{p}\right)$ where

(a) $a_{p} \in\left[\omega_{1}\right]^{<\omega}$

(b) $U_{p}: a_{p} \rightarrow C\left(\omega_{1}\right)$;

(c) for all $\alpha \in a_{p}$ we have $\operatorname{dom}\left(U_{p}(\alpha)\right) \subseteq\left[\gamma_{\alpha}, \gamma_{\alpha+1}\right)$;

(d) for all $\alpha<\beta$ such that $\alpha, \beta \in a_{p}$ we have $l_{\beta} \notin\left[U_{p}(\alpha)\right]_{\gamma_{\beta}}$;

(e) $2^{\omega_{1}}-\bigcup\left\{\left[U_{p}(\alpha)\right]: \alpha \in a_{p}\right\} \neq \emptyset$ (or equivalently, by (c), $U_{p}(\alpha) \neq \emptyset$, for all $\left.\alpha \in a_{p}\right)$.

We define the order as $p \leq q$ if and only if $a_{p} \supseteq a_{q}$ and $U_{p}\left\lceil a_{q}=U_{q}\right.$.

Lemma 5.4. Let $M$ be a model as in Section 2 such that $\omega_{1} \subseteq M$ and $\omega_{1} \in M$. Let $(\mathcal{M}, \mathcal{L})$ be as above and let $\mathcal{L} \subseteq M$. Suppose that for all $d \in C\left(\omega_{1}\right)$ the set $\left\{\alpha: l_{\alpha} \in[d]_{\gamma_{\alpha}}\right\}$ is uncountable and $P_{\mathcal{M}, \mathcal{L}}$ satisfies the c.c.c. Then $P_{\mathcal{M}, \mathcal{L}}$ forces that $2^{\omega_{1}} \cap M$ does not have the Lindelof property.

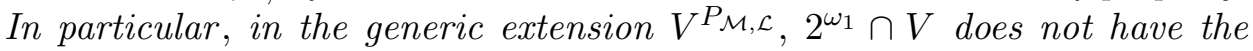
Lindelöf property.

Proof. Let $G \subseteq P$ be a $P$-generic over $V$. Let

$$
\mathcal{U}=\left\{\left[U_{p}(\alpha)\right]: p \in G \text { and } \alpha \in a_{p}\right\} .
$$

We will show that $\mathcal{U}$ is an open cover of $2^{\omega_{1}} \cap M$ without a countable subcover. 
Let $x \in 2^{\omega_{1}} \cap M$ and $q \in P$. We have to show that there are $p \leq q$ and $\alpha \in a_{p}$ such that $x \in\left[U_{p}(\alpha)\right]$. For this let $\gamma \in \omega_{1}$ be such that $\gamma \geq$ $\max \operatorname{dom} U_{q}\left(\alpha^{\prime}\right)$ for all $\alpha^{\prime} \in a_{q}$. Let $\alpha \in \omega_{1}$ be such that $\alpha>\gamma$ and

$$
l_{\alpha} \in 2^{\gamma_{\alpha}}-\bigcup\left\{\left[U_{q}\left(\alpha^{\prime}\right)\right]_{\gamma_{\alpha}}: \alpha^{\prime} \in a_{q}\right\} .
$$

We can find such an $\alpha$ using the assumption of the lemma and (e).

Now we define $p=\left(a_{p}, U_{p}\right)$ where $a_{p}=a_{q} \cup\{\alpha\}$ and

$$
U_{p}=U_{q} \cup\left\{\left\langle\alpha,\left\langle\gamma_{\alpha}, x\left(\gamma_{\alpha}\right)\right\rangle\right\rangle\right\} .
$$

It is clear that $p$ satisfies (a) and (b). Moreover, (c) is satisfied because $\operatorname{dom}\left(U_{p}(\alpha)\right)=\left\{\gamma_{\alpha}\right\}$. The condition (d) holds because $l_{\alpha} \in 2^{\gamma_{\alpha}}-\bigcup\left\{\left[U_{q}(\beta)\right]_{\gamma_{\alpha}}\right.$ : $\left.\beta \in a_{q}\right\}$ and finally (e) follows from the independence of $U_{p}(\alpha)$ from all $U_{q}(\beta)$ for $\beta \in a_{q}$.

Now we show that $\mathcal{U}$ does not have a countable subcover in the generic extension $V[G]$. Suppose that $\mathcal{V} \subseteq \mathcal{U} \subseteq C\left(\omega_{1}\right)$ is countable. Let $\alpha_{0}$ be a countable ordinal such that $\operatorname{supp}(U) \subseteq \alpha_{0}$ for all $U \in \mathcal{V}$. Since $P_{\mathcal{M}, \mathcal{L}}$ is c.c.c., we can decide $\alpha_{0}$ in the ground model. Now work in the ground model. As before it is easy to see that there is $p \in G$ such that $\max \left(a_{p}\right)>\alpha_{0}$. Let $\alpha \in a_{p}-\alpha_{0}$. By (d), this implies that $l_{\alpha} \notin U$ for all $U \in \mathcal{V}$. Let $l \in 2^{\omega_{1}}$ be in $M$ so that $l \mid \gamma_{\alpha}=l_{\alpha}$ (this follows from the fact that $l_{\alpha} \in M$ ). Now $l \notin \bigcup \mathcal{V}$ and so $\mathcal{V}$ is not a cover of $2^{\omega_{1}} \cap M$.

We will often be interested in some special pairs $(\mathcal{M}, \mathcal{L})$ introduced by the following definition:

Definition 5.5. Suppose that $\mathcal{M}=\left(M_{\alpha}\right)_{\alpha<\omega_{1}}$ and $\mathcal{L}=\left(l_{\alpha}\right)_{\alpha<\omega_{1}}$ are as before and let $M_{\alpha} \cap \omega_{1}=\gamma_{\alpha}$. We say that $(\mathcal{M}, \mathcal{L})$ is a Luzin pair if the following conditions are satisfied:

(f) for all $\alpha<\beta<\omega_{1}$ we have $\left|M_{\alpha}\right|=\omega, M_{\alpha} \in M_{\beta} \prec H\left(\omega_{1}\right)$ and $\bigcup_{\beta<\alpha} M_{\beta}=M_{\alpha}$ for $\alpha$ limit;

(g) $\bigcup \mathcal{M}=H\left(\omega_{1}\right)$;

(h) $l_{\alpha} \in M_{\alpha+1} \cap 2^{\gamma_{\alpha}}$;

(i) $l_{\alpha} \in \bigcap\left\{[D]_{\gamma_{\alpha}}: D \in M_{\alpha}, D \in\left[C\left(\gamma_{\alpha}\right)\right]^{\omega}, D\right.$ is predense in $\left.C\left(\omega_{1}\right)\right\}$;

(j) for all $d \in C\left(\omega_{1}\right)$, the set $\left\{\alpha: l_{\alpha} \in[d]_{\gamma_{\alpha}}\right\}$ is uncountable.

The point is that when $(\mathcal{M}, \mathcal{L})$ is a Luzin pair, $P_{\mathcal{M}, \mathcal{L}}$ has nice properties, for example it is c.c.c.

Lemma 5.6. Suppose $M$ is a model as in Section 2 such that $\omega_{1} \subseteq M$ and $2^{\omega} \cap M$ is not of the first Baire category in $2^{\omega}$. Suppose $C H$ holds. Then there is a Luzin pair $(\mathcal{M}, \mathcal{L})$ such that $\mathcal{L} \subseteq M$.

Proof. The construction of $M_{\alpha}$ and $l_{\alpha}$ is by induction on $\alpha<\omega_{1}$. Since $\omega_{1} \subseteq M$ and $\left|H\left(\omega_{1}\right)\right|=2^{\omega}$ (see [20], Exercise III 4), it is clear that $\mathrm{CH}$ provides the tools for the construction, in $M$, of $(\mathcal{M}, \mathcal{L})$ such that $(\mathrm{g}),(\mathrm{h})$ 
and (j) are satisfied. So let us focus on the construction of $l_{\alpha} \in M$, given $M_{\alpha}$, so that (i) is satisfied. In other words, we need to find $l_{\alpha} \in M$ which is in the intersection of a given countable collection of predense subsets of $C\left(\gamma_{\alpha}\right)$. Using the equivalence (in $M$, because $\omega_{1} \subseteq M$ ) of $2^{\omega}$ and $2^{\gamma_{\alpha}}$ we can work in $2^{\omega}$. Here, by our assumption, the intersection of any countable family of dense open sets must contain a point of $2^{\omega} \cap M$, so using the equivalence of $2^{\omega}$ and $2^{\gamma_{\alpha}}$ back we obtain the required $l_{\alpha}$.

Lemma 5.7. Suppose that $(\mathcal{M}, \mathcal{L})$ is a Luzin pair and let $D \subseteq C\left(\omega_{1}\right)$ be powerfully predense in $C\left(\omega_{1}\right)$. Let $\left(F_{\xi}\right)_{\xi<\omega_{1}}$ be a family of finite and pairwise disjoint subsets of $\omega_{1}$ of the same cardinality $n \in \omega$. Then

$$
\left\{\xi<\omega_{1}: \exists d \in D \forall \alpha \in F_{\xi} \quad l_{\alpha} \in[d]_{\gamma_{\alpha}}\right\}
$$

is co-countable.

Proof. Let $N$ be a countable elementary submodel of $H\left(\left(2^{\omega_{1}}\right)^{+}\right)$such that $D \in N,\left(F_{\xi}\right)_{\xi<\omega_{1}} \in N$ and $N \cap H\left(\omega_{1}\right)=M_{\alpha}$ for some $\alpha<\omega_{1}$ (the last property can be easily achieved, for example, if $\mathcal{M} \in N$ and taking $\left.\alpha=N \cap \omega_{1}\right)$. Using Fact 5.3, we can find $D_{1} \in[D]^{\omega}$ such that $D_{1}$ is powerfully predense in $C\left(\omega_{1}\right)$ and $D_{1} \in M_{\alpha}$. So without loss of generality we can assume that $D$ is countable and that it is an element of $M_{\alpha}$.

Let $\xi>\alpha$ and let $\alpha_{1}, \ldots, \alpha_{n}$ be elements of $F_{\xi}$ listed in increasing order. In particular $\alpha<\alpha_{i}$ for $i=1, \ldots, n$. Let us show that there is $d \in D$ such that $l_{\alpha_{i}} \in[d]_{\gamma_{\alpha_{i}}}$ for all $i=1, \ldots, n$. For this, we prove by induction on $0 \leq i<n$ that

$$
E_{i}=\left\{\langle d, \ldots, d\rangle \in \Delta_{n-i}(D): \forall j=1, \ldots, i l_{\alpha_{j}} \in[d]_{\gamma_{j}}\right\}
$$

is predense in $C\left(\gamma_{\alpha}\right)^{n-i}$. For $i=0$ this is just the fact that $\Delta_{n}(D)$ is predense in $C\left(\gamma_{\alpha}\right)^{n}$, which follows from the assumption that $D$ is powerfully predense in $C\left(\omega_{1}\right)$ and included in $C\left(\gamma_{\alpha}\right)$. So assume that we are done below $i<n$ and let us show that $E_{i}$ is predense in $C\left(\gamma_{\alpha}\right)^{n-i}$. Fix $d_{i+1}, \ldots, d_{n}$ in $C\left(\gamma_{\alpha}\right)$. The inductive hypothesis implies that

$$
D_{i}\left(d_{i+1}, \ldots, d_{n}\right)=\left\{d \in D: \forall j>i d \| d_{j} \text { and } \forall j<i l_{\alpha_{j}} \in[d]_{\gamma_{\alpha_{j}}}\right\}
$$

is predense in $C\left(\gamma_{\alpha}\right)$ (this is a straightforward consequence of the inductive assumption that $E_{i-1}$ is predense in $\left.C\left(\gamma_{\alpha}\right)^{n-(i-1)}\right)$. Thus, by Fact 5.2 , it is predense in $C\left(\gamma_{\alpha_{i}}\right)$. Also, the above set is an element of $M_{\alpha_{i}}$, so by (i) the element $l_{\alpha_{i}}$ is in $\left[D_{i}\left(d_{i+1}, \ldots, d_{n}\right)\right]_{\gamma_{\alpha_{i}}}$. This implies that there is $d \in$ $D_{i}\left(d_{i+1}, \ldots, d_{n}\right)$ such that $l_{\alpha_{i}} \in[d]_{\gamma_{\alpha_{i}}}$. But then this $d$ witnesses that there is an element of $E_{i}$ compatible with $\left\langle d_{i+1}, \ldots, d_{n}\right\rangle$, which completes the inductive step. In particular we have $E_{n-1}$ predense in $C\left(\gamma_{\alpha}\right)$ so, using (i) again, we conclude the lemma.

In what follows we will often use a result of Devlin from [5]. For the convenience of the reader we quote it below: 
Lemma 5.8 ([5], Corollary 3.15). Let B be a complete c.c.c. Boolean algebra. The following are equivalent:

(i) $B$ adds an $\omega_{1}$-branch;

(ii) there is a subset $T \subseteq B$ such that

(a) $\left\langle T, \geq_{B}\right\rangle$ is a Suslin tree and

(b) for each $\alpha<\omega_{1}, \operatorname{Lev}_{\alpha} T$ is a maximal antichain of $B$.

Proposition 5.9. Let $(\mathcal{M}, \mathcal{L})$ be a Luzin pair. Then

(1) $P_{\mathcal{M}, \mathcal{L}}$ is c.c.c.;

(2) $P_{\mathcal{M}, \mathcal{L}}$ does not add $\omega_{1}$-branches;

(3) $P_{\mathcal{M}, \mathcal{L}}$ has property (2) in all Cohen extensions.

Proof. Put $P=P_{\mathcal{M}, \mathcal{L}}$. First, let us see that (3) is satisfied if $P$ has property (2) in the forcing extension obtained by $C\left(\omega_{1}\right)$. For this, suppose that $P$ does not satisfy $(2)$ in the extension obtained by $C(\kappa)$ for some cardinal $\kappa>\omega_{1}$. By Lemma 5.8 , this means that there is a $C(\kappa)$-name $\dot{T}$ for a Suslin tree so that, in $V^{C(\kappa)}$, forcing with $P$ adds an $\omega_{1}$-branch in $T$, where $T$ is the value of the name $\dot{T}$. Because the cardinality of $T$ is $\omega_{1}$, we deduce that $T \in V^{C(A)}$ for some $A \subseteq \kappa$ of cardinality $\omega_{1}$, where $A$ is in the ground model. In $V^{C(A) \times C(\kappa-A) \times P}$ the tree $T$ has an $\omega_{1}$-branch. As $V^{C(A) \times C(\kappa-A) \times P}=V^{C(A) \times P \times C(\kappa-A)}$ and $C(\kappa-A)$ does not add $\omega_{1}$-branches (because, for example, by 5.8 it would contradict the precaliber $\omega_{1}$ of the Cohen forcing), $T$ has an $\omega_{1}$-branch in $V^{C(A) \times P}$. But $T$ does not have an $\omega_{1}$-branch in $V^{C(A)}$ because it is a Suslin tree in $V^{C(\kappa)}$. In other words, $C(A)$, which is equivalent to $C\left(\omega_{1}\right)$, destroys property (2) of $P$.

To show (1), (2), and (3) restricted to $C\left(\omega_{1}\right)$ it is enough to prove the following:

Claim. Let $\dot{T}$ be a $C\left(\omega_{1}\right)$-name for a Suslin tree and $\left(\sigma_{\alpha}, \dot{t}_{\alpha}, p_{\alpha}\right)_{\alpha<\omega_{1}}$ be such that $\sigma_{\alpha} \in C\left(\omega_{1}\right), \sigma_{\alpha} \Vdash \dot{t}_{\alpha} \in \operatorname{Lev}_{\alpha}(\dot{T})$ and $p_{\alpha} \in P$ for all $\alpha<\omega_{1}$. Then there are $\alpha, \beta \in \omega_{1}, \alpha<\beta$, such that $\sigma_{\alpha} \cup \sigma_{\beta} \in C\left(\omega_{1}\right), p_{\alpha} \| p_{\beta}$ and

$$
\sigma_{\alpha} \cup \sigma_{\beta} \| \dot{t}_{\alpha} \leq_{\dot{T}} \dot{t}_{\beta} \text {. }
$$

First, let us prove that the proposition follows from the claim. Clearly (1) would be proved because we can choose $\sigma_{\alpha}=\emptyset$. Now let us see that, assuming (3) and (1), the negation of (2) leads to a contradiction. Clause (1) and the negation of (2), by 5.8, imply that there is a Suslin tree with predense levels included in the completion of $P$. Note that Cohen forcing preserves this Suslin tree as well as the c.c.c. of $P$ and so (3) cannot hold in the Cohen extension. Finally, assume the claim and that (3) is false and let us show we get a contradiction. Using again 5.8 , we can assume that there is a $C\left(\omega_{1}\right)$-name $\dot{T}$ for a Suslin tree and a $C\left(\omega_{1}\right) \times P$-name $\dot{b}$ for an $\omega_{1}$-branch of $\dot{T}$. 
Choose $\sigma_{\alpha}, p_{\alpha}$ and $\dot{t}_{\alpha}$ so that

$$
\left(\sigma_{\alpha}, p_{\alpha}\right) \Vdash \dot{t}_{\alpha}=\dot{b}(\alpha) .
$$

Let $\alpha, \beta$ be as in the claim, $\sigma \leq \sigma_{\alpha} \cup \sigma_{\beta}$ be such that $\sigma \Vdash \dot{t}_{\alpha} \mathbb{E}_{\dot{T}} \dot{t}_{\beta}$ and $p \in P$ be such that $p \leq p_{\alpha}, p_{\beta}$. Now

$$
(\sigma, p) \Vdash \dot{b}(\alpha) \underline{\leq}_{\dot{T}} \dot{b}(\beta),
$$

a contradiction.

Proof of the claim. Let $\dot{T}$ and $\left(\sigma_{\alpha}, \dot{t}_{\alpha}, p_{\alpha}\right)_{\alpha<\omega_{1}}$ be as in the assumptions of the claim. Let $N$ be a countable elementary submodel of $H\left(\omega_{2}\right)$ such that all relevant objects are in $N$ and such that there is an $\alpha_{0}<\omega_{1}$ with $N \cap H\left(\omega_{1}\right)=M_{\alpha_{0}}$ and $\alpha_{0}=\gamma_{\alpha_{0}}$ (again, $\mathcal{M} \in N$ would imply it for $\alpha_{0}=$ $\left.N \cap \omega_{1}\right)$.

Let $\xi<\omega_{1}$ be such that $\xi>\gamma_{\alpha_{0}}, a=a_{p_{\xi}} \cap \gamma_{\alpha_{0}}$ and $\mathcal{U}=\mathcal{U}_{p_{\xi}} \mid a$. Let $m_{0} \in \omega$ be the cardinality of $a_{p_{\xi}}$. Using the definition of $P$, it is easy to see that $(a, \mathcal{U})$ is a condition of $P$ in $M_{\alpha_{0}}$ and that $p_{\xi} \leq(a, \mathcal{U})$.

Let $\sigma=\sigma_{\xi} \mid \alpha_{0}$. Note that for all $\eta<\gamma_{\alpha_{0}}$ such that $\eta>\max a, \max \operatorname{dom}(\sigma)$, the structure $H\left(\omega_{2}\right)$ satisfies the formula

$$
\exists \xi>\eta \quad p_{\xi} \leq(a, \mathcal{U}), a_{p_{\xi}} \cap \eta=a, \sigma=\sigma_{\xi}|\eta,| a_{p_{\xi}} \mid=m_{0} .
$$

So by the elementarity of the model $N$, by induction and by the $\Delta$-system lemma (inside $N$ ) the model $N$ satisfies the following: there is a strictly increasing sequence $\left\{\delta_{\xi}: \xi<\omega_{1}\right\}$ and sequences $\left\{p_{\xi}: \xi<\omega_{1}\right\}$ in $P$ and $\left\{\sigma_{\xi}: \xi<\omega_{1}\right\}$ in $C\left(\omega_{1}\right)$ such that $p_{\xi} \leq(a, \mathcal{U}), a_{p_{\xi}} \cap \delta_{\xi}=a, a_{p_{\xi}}-\delta_{\xi} \subseteq \delta_{\xi+1}$, $\sigma=\sigma_{\xi} \mid \delta_{\xi}, \sigma_{\xi}-\sigma \in C\left(\delta_{\xi+1}-\delta_{\xi}\right)$ and $\left|a_{p_{\xi}}\right|=m_{0}$ for all $\xi<\omega_{1}$.

Let us see that this implies that $\mathcal{F}$ is powerfully predense in $C\left(\omega_{1}\right)$ where

$$
\begin{aligned}
& \mathcal{F}=\left\{d \in C\left(\omega_{1}\right): \exists \xi_{1}<\xi_{2}<\omega_{1} \quad p_{\xi_{1}}, p_{\xi_{2}} \leq(a, \mathcal{U}),\right. \\
& {[d] \subseteq 2^{\omega_{1}}-\left[\left\{U_{p_{\xi_{1}}}(\alpha): \alpha \in a_{p_{\xi_{1}}}-a\right\} \cup\left\{U_{p_{\xi_{2}}}(\alpha): \alpha \in a_{p_{\xi_{2}}}-a\right\}\right], } \\
&\left.\sigma \cup \sigma_{\xi_{1}} \cup \sigma_{\xi_{2}} \in C\left(\omega_{1}\right), \sigma \cup \sigma_{\xi_{1}} \cup \sigma_{\xi_{2}} \forall \forall \dot{t}_{\xi_{1}} \leq_{\dot{T}} \dot{t}_{\xi_{2}}\right\} .
\end{aligned}
$$

To see this, choose $d_{1}, \ldots, d_{n} \in C\left(\omega_{1}\right)$. We can find $\xi_{0}$ such that $\operatorname{dom}\left(d_{i}\right) \subseteq$ $\delta_{\xi_{0}}$ for all $i=1, \ldots, n$. By the independence of the elements of $C\left(\omega_{1}\right)$ with disjoint domains, it is clear that for any $\xi_{1}>\xi_{2}>\xi_{0}$ we have

$$
\left[d_{i}\right] \cap\left(2^{\omega_{1}}-\left[\left\{U_{p_{\xi_{1}}}(\alpha): \alpha \in a_{p_{\xi_{1}}}-a\right\} \cup\left\{U_{p_{\xi_{2}}}(\alpha): \alpha \in a_{p_{\xi_{2}}}-a\right\}\right]\right) \neq \emptyset .
$$

Also $\sigma_{\xi_{1}} \cup \sigma_{\xi_{2}} \in C\left(\omega_{1}\right)$. So the above complements are good candidates for $d$ and really to see that $\mathcal{F}$ is powerfully predense we only need to find two distinct $\xi_{0}<\xi_{1}<\xi_{2}$ such that $\sigma_{\xi_{1}} \cup \sigma_{\xi_{2}} \Vdash \dot{t}_{\xi_{1}} \leq \dot{t}_{\xi_{2}}$. But if we cannot find $\xi_{1}, \xi_{2}$ with these properties, it means that all pairs satisfy the negation of this property. As $\sigma$ forces that for uncountably many $\xi$ 's the conditions $\sigma_{\xi}$ are in the $C\left(\omega_{1}\right)$-generic set, this would mean that $\sigma$ forces that

$$
\left\{\dot{t}_{\delta_{\xi}}: \sigma_{\xi} \in \Gamma\right\}
$$


is an uncountable branch in $\dot{T}$, where $\Gamma$ is the canonical $C\left(\omega_{1}\right)$-name for the generic set. But this contradicts the fact that $C\left(\omega_{1}\right)$ forces that $\dot{T}$ is a Suslin tree, which implies that $\mathcal{F}$ is powerfully predense in $C\left(\omega_{1}\right)$.

Using Fact 5.3, we can find $D \in[\mathcal{F}]^{\omega}$ such that $D$ is powerfully predense in $C\left(\omega_{1}\right)$ and that $D \in M_{\alpha_{0}}$. Now using Lemma 5.7 (in $N$ ), for $D$ and $F_{\xi}=a_{p_{\xi}}-a$, we can find in $N$ (and therefore in $M_{\alpha_{0}}$ ) the upper limit of the complement of the co-countable set mentioned in the lemma. Let $\eta<\omega_{1}$ be outside $M_{\alpha_{0}}$ and so above this limit. The lemma implies that there is $d \in D$ such that $l_{\alpha} \in[d]_{\gamma_{\alpha}}$ for all $\alpha \in a_{p_{\eta}}-a$. Since $d \in D \in M_{\alpha_{0}}$ and $D \subseteq \mathcal{F}$, the ordinals $\xi_{1}, \xi_{2}$ mentioned in the definition of $\mathcal{F}$ are in $M_{\alpha_{0}}$, so this also applies to derived objects like $\sigma_{\xi_{1}}, p_{\xi_{2}}$ etc. This in turn implies that $p_{\xi_{i}} \| p_{\eta}$ and $\sigma_{\eta} \cup \sigma_{\xi_{i}} \in C\left(\omega_{1}\right)$ for $i=1$, 2. (For the compatibility, note that for every $\alpha \in a_{p_{\eta}}-a$, we have $l_{\alpha} \in[d]_{\gamma_{\alpha}}$ and $[d] \cap\left[U_{p_{\xi_{i}}}\left(\alpha^{\prime}\right)\right]=\emptyset$, for every $\alpha^{\prime} \in a_{p_{\xi_{i}}}-a$.) So to finish the proof of the claim we only need to find $i \in\{1,2\}$ so that $\sigma_{\eta} \cup \sigma_{\xi_{i}} \| \dot{t}_{\xi_{i}} \leq_{\dot{T}} \dot{t}_{\eta}$.

As $\sigma_{\xi_{1}} \cup \sigma_{\xi_{2}} \forall \dot{t}_{\xi_{1}} \leq_{\dot{T}} \dot{t}_{\xi_{2}}$ and $\sigma_{\xi_{1}}, \sigma_{\xi_{2}} \in M_{\alpha_{0}}$, there is $\sigma^{\prime} \leq \sigma_{\xi_{1}}, \sigma_{\xi_{2}}$ in $C\left(\alpha_{0}\right)$ such that $\sigma^{\prime} \Vdash \dot{t}_{\xi_{1}} \not \leq \dot{t}_{\xi_{2}}$.

As $\sigma^{\prime} \in C\left(\alpha_{0}\right)$ and $\sigma_{\eta}\left\lceil\alpha_{0}=\sigma\right.$ we have $\sigma^{\prime} \cup \sigma_{\eta} \in C\left(\omega_{1}\right)$. Now let $\varrho \leq \sigma^{\prime} \cup \sigma_{\eta}$ and $n_{1}, n_{2}, n_{3}$ be such that

$$
\varrho \Vdash \dot{t}_{\xi_{1}} \leq_{\dot{T}} \dot{t}_{\alpha_{0}, n_{1}}, \dot{t}_{\xi_{2}} \leq_{\dot{T}} \dot{t}_{\alpha_{0}, n_{2}}, \dot{t}_{\alpha_{0}, n_{3}} \leq_{\dot{T}} \dot{t}_{\eta},
$$

where $\left\{\dot{t}_{\alpha_{o}, n}: n \in \omega\right\}$ are names for all distinct elements of $\operatorname{Lev}_{\alpha_{0}}(\dot{T})$. Now as $\varrho \Vdash \dot{t}_{\xi_{1}} \not \leq \dot{t}_{\xi_{2}}$, we find that $n_{1} \neq n_{2}$ and then we have $i \in\{1,2\}$ such that $n_{i} \neq n_{3}$. Thus $\varrho \Vdash \dot{t}_{\xi_{i}} \mathbb{Z}_{\dot{T}} \dot{t}_{\eta}$ and so $\sigma_{\xi_{i}} \cup \sigma_{\eta} \Vdash \dot{t}_{\xi_{i}} \leq \dot{t}_{\eta}$, which completes the proof of the claim.

Corollary 5.10. ( $\mathrm{CH})$ Suppose $M$ is a model as in Section 2 such that $2^{\omega} \cap M$ is of the second Baire category. Then there is a c.c.c. forcing $P$ which does not add $\omega_{1}$-branches and $P$ forces that $2^{\omega_{1}} \cap M$ does not have the Lindelöf property.

Proof. Use Lemmas 5.4 and 5.6 and Proposition 5.9.

We do not really understand the relation between the category of $M \cap 2^{\omega_{1}}$ and the possibility of destroying the Lindelöf property of $M \cap 2^{\omega_{1}}$ by a c.c.c. forcing. For example, after adding many random reals $\mathrm{CH}$ may fail and $V \cap 2^{\omega_{1}}$ is of the first category, but the forcing $P_{\mathcal{M}, \mathcal{L}}$ which destroys the Lindelöf property of $V \cap 2^{\omega_{1}}$ (which exists before adding the random reals) stays c.c.c. (use the fact that the random forcing is $\sigma$-linked; [1], Lemma 3.1.1). This is also related to Question 1. We can also obtain the following corollary:

COROLlaRY 5.11. There is a proper forcing which does not add $\omega_{1}$ branches and forces that $2^{\omega_{1}} \cap V$ does not have the Lindelöf property. 
Proof. The forcing is $P=C\left(\omega_{2}\right) * \operatorname{Coll} * P_{\mathcal{M}, \mathcal{L}}$ where Coll is the $C\left(\omega_{2}\right)$ name for the $\sigma$-closed collapse of $2^{\omega}$ (in $V^{C\left(\omega_{2}\right)}$ ) onto $\omega_{1}$. Note that $\mathrm{CH}$ holds in $V^{C\left(\omega_{2}\right) * \text { Coll }}$ and $V \cap 2^{\omega}$ is not of the first category as the Cohen forcing preserves second category of the old continuum (see [1], Lemma 3.3.17) and Coll does not add new codes for Borel subsets of $2^{\omega}$. So by Lemma 5.6 we have a Luzin pair $(\mathcal{M}, \mathcal{L}), \mathcal{L} \subseteq V$, and therefore $P_{\mathcal{M}, \mathcal{L}}$ has the properties stated in Proposition 5.9 in $V^{C\left(\omega_{2}\right) * \text { Coll }}$. It is not difficult to see that $C\left(\omega_{2}\right) *$ Coll does not add branches (e.g. use the same argument as for the Mitchell forcing $M i$, see e.g. [29], Lemma 1.5). So Proposition 5.9(b) implies that $P$ does not add uncountable branches. On the other hand, Lemma 5.4 implies that $2^{\omega_{1}} \cap V$ is not Lindelöf in $V^{P}$.

On the other hand, recall our result from the previous section which says that it is independent that there is a c.c.c. forcing destroying the Lindelöf property of a compact space. The following observation shows some ZFC link between adding $\omega_{1}$-branches and destroying the Lindelöf property.

FACT 5.12. Suppose that $P$ is a forcing which does not collapse $\omega_{1}$ but adds an $\omega_{1}$-branch. Then $P$ destroys the Lindelöf property of a compact space.

Proof. Without loss of generality we can assume that $P$ is a complete Boolean algebra. Let $X$ be the Stone space of $P$. Let $\dot{b}$ be a $P$-name for the new uncountable branch of a tree $T$ of height $\omega_{1}$. Consider the following conditions of $P$ or clopen subsets of $X$ :

$$
p(\alpha, t)=\sup \{p: p \Vdash \dot{b}(\alpha)=t\} .
$$

Work in $V^{P}$ and let $b$ be the value of the name $\dot{b}$. Consider the open cover of $X$ given by $\left\{X-p(\alpha, b(\alpha)): \alpha<\omega_{1}\right\}$. This family covers $X$ because any point $x \in X$ missed by the family would allow the definition

$$
\left\{t \in T: \exists \alpha<\omega_{1} \quad x \in p(\alpha, t)\right\}
$$

in $V$. But the above set is equal to $b$, which is impossible as $b \notin V$. Also note that the cover cannot have a countable subcover. This is because it is a nondecreasing sequence of clopen sets. So a countable subcover would imply that the sequence stabilizes from some point on (use the fact that $\omega_{1}$ is not collapsed), e.g. $X-p(\alpha, b(\alpha))$. This would mean that $p(\alpha, b(\alpha))$ decides the entire branch $b$, which again contradicts the fact that $b \notin V$.

Finally let us make use of Proposition 5.9 for a consistency result concerning the families $\left[2^{\omega_{1}}\right]_{L}^{\omega_{1}}$ and $\left[2^{\omega_{1}}\right]_{0}^{\omega_{1}}$. First we need the following:

Lemma 5.13. Suppose $P$ is a forcing notion which is c.c.c. and does not add $\omega_{1}$-branches and for every $\alpha$ the forcing $C(\alpha)$ forces that $P$ does not add $\omega_{1}$-branches. Let $S$ be a forcing notion for which there is an $S$-name $\dot{R}$ for a forcing notion such that $S * \dot{R}$ is equivalent to $C\left(\omega_{1}\right) *\left(\dot{Q} \times C\left(\kappa-\omega_{1}\right)\right)$ 
for some cardinal $\kappa$, where $\dot{Q}$ is a $C\left(\omega_{1}\right)$-name for a $\sigma$-closed forcing. Then in $V^{S}$ the forcing $P$ satisfies the c.c.c. and does not add $\omega_{1}$-branches.

Proof. First note that $P$ satisfies the c.c.c. in $V^{S}$. Suppose not. Then an uncountable antichain of $P$ in $V^{S}$ would stay uncountable in $V^{S * \dot{R}}$ ( $R$ must preserve $\omega_{1}$ as $C\left(\omega_{1}\right) *\left(\dot{Q} \times C\left(\kappa-\omega_{1}\right)\right)$ which is equivalent to $S * \dot{R}$ is proper $)$. This would mean that $C\left(\omega_{1}\right) *\left(\dot{Q} \times C\left(\kappa-\omega_{1}\right)\right)$ adds an uncountable antichain in $P$. But Cohen forcings cannot add one because they have precaliber $\omega_{1}$, and $\sigma$-closed forcings cannot add one either because we could decide some uncountable antichain in $P$ in the ground model, so we have obtained a contradiction.

Now let us see that $P$ does not add $\omega_{1}$-branches in $V^{S}$. Suppose it does. By 5.8, this means that in $V^{S}$ there is a Suslin tree in the completion of $P$ with predense levels (we already know that $P$ is c.c.c. in $V^{S}$, so we can use 5.8). Note that this Suslin tree stays Suslin in any extension that preserves $\omega_{1}$ and preserves the c.c.c. of $P$. The density of the levels is also not affected. Using the proof of the first part, we know that $R$ preserves $\omega_{1}$ and preserves the c.c.c. of $P$, thus there is a Suslin tree with predense levels in the completion of $P$ after forcing with $P * \dot{R}$ which is equivalent to $C\left(\omega_{1}\right) *\left(\dot{Q} \times C\left(\kappa-\omega_{1}\right)\right)$. But by the assumption about $P$ the forcing $C\left(\omega_{1}\right)$ does not add such a tree and $Q$ preserves the property that the $P$ does not add $\omega_{1}$-branches in any Cohen extension, as any $Q$-name for a $C\left(\kappa-\omega_{1}\right)$-name for a Suslin subtree of $P$ with dense levels would give rise to a $C\left(\kappa-\omega_{1}\right)$-name for a Suslin subtree of $P$ with dense levels, so we have obtained a contradiction.

THEOREM 5.14. Assume the consistency of ZFC together with the existence of a Mahlo cardinal. Then it is consistent (with $Z F C+\neg w K H+M A$ ) that $0 \leq\left[2^{\omega_{1}}\right]_{L}^{\omega_{1}}<\left[2^{\omega_{1}}\right]_{0}^{\omega_{1}}<1$.

Proof. In [29] the construction of a model of $\mathrm{MA}+\neg \mathrm{CH}+\neg \mathrm{KH}$ of K. Devlin [5] was enriched and MA $+\neg \mathrm{wKH}$ was shown to hold in any model of the following form:

We start with any model of GCH with an inaccessible (or larger cardinal if needed), we force with the Mitchell forcing $M i_{\kappa}$ and then we force with an iteration $Q_{\kappa}$. In $V^{M i_{\kappa}}$ we have $\kappa=\omega_{2}$ and $\left(Q_{\alpha}, \dot{Q}^{\alpha}\right)_{\alpha \leq \kappa}$ is an iteration with finite supports of c.c.c. forcings such that $Q_{\alpha}$ forces that $\dot{Q}^{\alpha}$ is a c.c.c. forcing of cardinality $\omega_{1}$ which does not add $\omega_{1}$-branches. The forcings $\dot{Q}^{\alpha}$ can be arranged as we wish as long as the final model satisfies MA. For this purpose we have to consider all c.c.c. forcings of size $\omega_{1}$. By the result of Devlin (Lemma 5.8) we can restrict our attention to forcings of size $\omega_{1}$ which do not add $\omega_{1}$-branches (because we can kill the c.c.c.-ness of forcings without this property with forcings with this property (see [5])). 
We will prove the theorem considering a model obtained by a forcing as above with some additional properties. One of them will be that $\kappa$ is a Mahlo cardinal (see [14]), i.e., $S=\{\alpha<\kappa: \alpha$ is inaccessible $\}$ is a stationary set in $\kappa$. To express other properties, we need the following claims in which the iteration $Q_{\kappa}$ is any iteration which satisfies the above description.

Claim. There is a club set $C \subseteq \kappa$ such that for every $\alpha \in C$ we have $M i_{\kappa} \Vdash Q_{\alpha} \in V^{M i_{\alpha}}$.

Proof. This follows from $\kappa$-c.c. of the forcing $M i_{\kappa}$ (see (v) of Section 4) and the fact that in $V^{M i_{\kappa}}$ the forcings $Q_{\alpha}$ for $\alpha<\kappa$ have cardinality less than $\kappa$.

Claim. Let $C \subseteq \kappa$ be as in the claim above. Then for $\gamma \in C$ the forcing $M i_{\gamma} * Q_{\gamma}$ is completely embedded in $M i_{\kappa} * Q_{\kappa}$.

Proof. Use the product lemma (see [20]) in $V^{M i_{\gamma}}$ for $M i_{[\gamma, \kappa)}$ and $Q_{\gamma}$.

Claim. Let $C \subseteq \kappa$ be as above. Suppose that $\gamma \in C \cup\{\kappa\}$ is an inaccessible cardinal which is a limit point of $C \cup\{\kappa\}$. Then

$$
\left[2^{\omega_{1}}\right]^{\omega_{1}} \cap V^{M i_{\gamma} * Q_{\gamma}}=\bigcup_{\beta \in C \cap \gamma}\left[2^{\omega_{1}}\right]^{\omega_{1}} \cap V^{M i_{\beta} * Q_{\beta}} .
$$

Proof. Use the fact that $M i_{\gamma}$ satisfies the $\gamma$-c.c. (by (v), Section 4) and that the supports of the conditions from $M i_{\gamma}$ have cardinality less than $\gamma$ (they are countable).

As in Section 4, we define by induction, in $V$, a sequence $\left(\dot{X}_{\alpha}: \alpha \in \kappa\right)$ of $M i_{\kappa}$-names for subsets of $2^{\omega_{1}}$ of cardinality $\omega_{1}$ and a sequence of ordinals $\left(\gamma_{\alpha}: \alpha<\kappa\right) \subseteq C$ in the following way: $M i_{\kappa} \Vdash \dot{X}_{\alpha}=\bigcup_{\beta<\alpha} \dot{X}_{\beta}$ and $\gamma_{\alpha}=$ $\sup \left(\gamma_{\beta}: \beta<\alpha\right)$ for $\alpha$ limit and if $\alpha=\beta+1$ for some $\beta$ we require that $M i_{\kappa} \Vdash \dot{X}_{\alpha}=2^{\omega_{1}} \cap V^{M i_{\gamma_{\alpha}} * Q_{\gamma_{\alpha}}}$ for $\gamma_{\alpha} \in C$ minimal greater than $\gamma_{\beta}$ such that $M i_{\kappa} \Vdash \dot{X}_{\beta} \subset V^{M i_{\gamma_{\alpha}} * Q \gamma_{\alpha}}$; this $\gamma_{\alpha}$ exists by the first claim above.

We are now ready to specify our iteration. We consider $\kappa$ which is a Mahlo cardinal. The forcings $Q^{\alpha}$ 's are arranged so that in $V^{M i_{\kappa} * Q_{\alpha}}$ we have for $\alpha \in \kappa$ :

(1) $\dot{Q}^{\alpha}$ has the c.c.c.

(2) $\dot{Q}^{\alpha}$ has cardinality $\omega_{1}$.

(3) $\dot{Q}^{\alpha}$ does not add $\omega_{1}$-branches.

and additionally:

(4) MA holds in the final model.

(5) For $\alpha \in C$ we define $\dot{Q}^{\alpha}$ in $V^{M i_{\kappa} * Q_{\alpha}}$ to be the forcing of the form $P_{\mathcal{M}, \mathcal{L}}$ for some pair $(\mathcal{M}, \mathcal{L})$ such that $\mathcal{L} \subseteq 2^{\omega_{1}} \cap V^{M i_{\alpha} * Q_{\alpha}}$. Thus we need the following: 
Claim. Let $\alpha \in C$. There exists in $V^{M i_{\kappa} * Q_{\alpha}}$ a pair $(\mathcal{M}, \mathcal{L})$ such that $\mathcal{L} \subseteq 2^{\omega_{1}} \cap V^{M i_{\alpha} * Q_{\alpha}}$ and $M i_{\kappa} * Q_{\alpha} \Vdash(1)$, (2) and (3) are satisfied for $\dot{Q}^{\alpha}=P_{\mathcal{M}, \mathcal{L}}$.

Proof. We have $2^{\omega}=\alpha$ in $V^{M i_{\alpha+1}}$. Now, the forcing $M i^{\alpha+2}$ is a collapse of $\alpha$ onto $\omega_{1}$ which does not add new reals, i.e., $V^{M i_{\alpha+2}}$ satisfies the $\mathrm{CH}$ and so $V^{M i_{\alpha+2} * Q_{\alpha}}$ satisfies the $\mathrm{CH}$ by (2) above. Also note that $2^{\omega} \cap V^{M i_{\alpha} * Q_{\alpha}}$ is not of the first Baire category in $V^{M i_{\alpha+2} * Q_{\alpha}}$. This follows from the fact that $M i_{\alpha+2} * Q_{\alpha}$ is equivalent to $M i_{\alpha} * Q_{\alpha} \times C(\omega) * \operatorname{Coll}(\alpha)$, where $\operatorname{Coll}(\alpha)$ is a $\sigma$-closed collapse of $\alpha$ onto $\omega_{1}$, and the fact that the Cohen forcing preserves the second category of the old continuum (see [1], Lemma 3.3.17) and that the collapse does not add codes for new Borel sets. This means that we can apply Lemma 5.6 to get the existence of a $\operatorname{Luzin}$ pair $(\mathcal{M}, \mathcal{L})$ in $V^{M i_{\alpha+2} * Q_{\alpha}}$ such that $\mathcal{L} \subseteq 2^{\omega_{1}} \cap V^{M i_{\alpha} * Q_{\alpha}}$. Now by 5.9 , the forcing $P_{\mathcal{M}, \mathcal{L}}$ is c.c.c. and does not add $\omega_{1}$-branches and clearly is of cardinality $\omega_{1}$ (as $\mathrm{CH}$ holds) in $V^{M i_{\alpha+2} * Q_{\alpha}}$. We need to see that these properties are also satisfied in $V^{M i_{\kappa} * Q_{\alpha}}$. For this we use properties (ii) and (iv), from Section 4, of the forcing $M i_{\kappa}$ combined with $5.9(3)$ and the conclusion follows from 5.13, so the claim is proved.

Now let us prove that the statement of the theorem holds in $V^{M i_{\kappa} * Q_{\kappa}}$. We will denote by $C^{\circ}$ the set of limit points of $C$. Clearly $\left(X_{\alpha}: \alpha \in \kappa\right)$ is an increasing club sequence in $\left[2^{\omega_{1}}\right]^{\omega_{1}}$ and so $\left(X_{\alpha}: \gamma_{\alpha} \in C^{\circ} \cap S\right)$ forms a stationary set in $\left[2^{\omega_{1}}\right]^{\omega_{1}}$ ( $S$ is stationary in $V$ and by the properness of the forcing $M i_{\kappa} * Q_{\kappa}$ it stays stationary in $\left.V^{M i_{\kappa} * Q_{\kappa}}\right)$. As the iteration does not add $\omega_{1}$-branches (see [5]) by the second claim we find that $X_{\alpha} \in\left[2^{\omega_{1}}\right]_{0}^{\omega_{1}}$ for all $\gamma_{\alpha} \in C^{\circ} \cap S$. On the other hand $\left\{X_{\alpha}: \gamma_{\alpha} \in C^{\circ} \cap S\right\}$ is disjoint from $\left[2^{\omega_{1}}\right]_{L}^{\omega_{1}}$. This follows from Lemma $5.4 \mathrm{in} V^{M i_{\alpha+2} * Q_{\alpha+1}}$ and the fact that not being Lindelöf is preserved by proper forcings (this follows from the fact that if $P$ is a proper forcing and $A$ is a countable set of ordinals in $V^{P}$, then there is a countable set of ordinals $B$ in $V$ such that $A \subseteq B$ ). This completes the proof of the theorem.

Corollary 5.15. Assume the consistency of the existence of a Mahlo cardinal. It is consistent that there is a model $M$ as in Section 2 such that $M \cap 2^{\omega_{1}}$ is not Lindelöf but there is no one-point $K$ which witnesses the failure of 2.4 .

Proof. Let $M \cap 2^{\omega_{1}} \in\left[2^{\omega_{1}}\right]_{o}^{\omega_{1}} \backslash\left[2^{\omega_{1}}\right]_{L}^{\omega_{1}}$. The one-point $K$ as in 2.4 would give rise to a convergent sequence in $M$ with limit outside $M$, which would contradict the choice of $M$.

Corollary 5.16. Assume the consistency of the existence of a Mahlo cardinal. It is consistent that there is a dense subgroup $X \subseteq 2^{\omega_{1}}$ and a compact set $K \subseteq 2^{\omega_{1}}$ such that $K \cap X=\emptyset$, there is no $G_{\delta}$-set $G$ such that 
$K \subseteq G$ and $X \cap G=\emptyset$ but for each $y \in K$ there is a $G_{\delta}$-set $G$ such that $y \in G$ and $G \cap X=\emptyset$.

Proof. Let $X=2^{\omega_{1}} \cap M \in\left[2^{\omega_{1}}\right]_{0}^{\omega_{1}} \backslash\left[2^{\omega_{1}}\right]_{L}^{\omega_{1}}$.

\section{References}

[1] T. Bartoszyński and H. Judah, Set Theory. On the Structure of the Real Line, A. K. Peters, Wellesley, MA, 1995.

[2] J. Barwise, M. Kaufmann, and M. Makkai, Stationary logic, Ann. Math. Logic 13 (1978), 171-224.

[3] J. Baumgartner, Applications of the proper forcing axiom, in: Handbook of SetTheoretic Topology, North-Holland, Amsterdam, 1984, 913-959.

[4] J. E. Baumgartner and F. D. Tall, Reflecting Lindelöfness, Topology Appl., to appear.

[5] K. Devlin, $\aleph_{1}$-trees, Ann. Math. Logic 13 (1978), 267-330.

[6] A. Dow, An empty class of nonmetric spaces, Proc. Amer. Math. Soc. 104 (1988), 999-1001.

[7] -, An introduction to applications of elementary submodels to topology, Topology Proc. 13 (1988), 17-72.

[8] -, Two applications of reflection and forcing to topology, in: Proc. Sixth Prague Topological Symposium, Z. Frolík (ed.), Heldermann, Berlin, 1988, 155-172.

[9] P. Erdős, A. Hajnal, and E. Milner, On the complete subgraphs of graphs defined by systems of sets, Acta Math. Hungar. 17 (1966), 159-229.

[10] R. Grunberg, L. R. Junqueira, and F. D. Tall, Forcing and normality, Topology Appl. 84 (1998), 145-174.

[11] A. Hajnal and I. Juhász, On hereditarily $\alpha$-Lindelöf and $\alpha$-separable spaces II, Fund. Math. 81 (1974), 174-158.

[12] -, Remarks on the cardinality of compact spaces and their Lindelöf subspaces, Proc. Amer. Math. Soc. 59 (1976), 146-148.

[13] T. Jech, Some combinatorial problems concerning uncountable cardinals, Ann. Math. Logic 5 (1973), 165-198.

[14] —, Set Theory, Academic Press, New York, 1978.

[15] I. Juhász, lecture, 1996 Spring Topology Conference.

[16] -, Cardinal Functions in Topology: Ten Years Later, Math. Center Tracts, 1980.

[17] L. R. Junqueira, Preservation of topological properties by elementary submodels and by forcing, Ph.D. thesis, Univ. of Toronto, 1996.

[18] L. R. Junqueira and F. D. Tall, The topology of elementary submodels, Topology Appl. 82 (1998), 239-266.

[19] D. Kueker, Countable approximations and Löwenheim-Skolem theorems, Ann. Math. Logic 11 (1977), 57-103.

[20] K. Kunen, Set Theory: An Introduction to Independence Proofs, North-Holland, Amsterdam, 1980.

[21] N. Luzin, Sur un problème de M. Baire, C. R. Hebdomadaires Séances Acad. Sci. Paris 158 (1914), 1258-1261.

[22] W. Mitchell, Aronszajn trees and the independence of the transfer property, Ann. Math. Logic 5 (1972/73), 21-46.

[23] S. Shelah, A compactness theorem for singular cardinals, free algebras, Whitehead problem and transversals, Israel J. Math. 21 (1975), 319-349. 
[24] S. Shelah, Generalized quantifiers and compact logic, Trans. Amer. Math. Soc. 204 (1975), 342-364.

[25] - On uncountable Boolean algebras with no uncountable pairwise comparable or incomparable sets of elements, Notre Dame J. Formal Logic 22 (1981), 301-308.

[26] —, On some problems in general topology, in: Set Theory (Boise, ID, 1992-1994), Amer. Math. Soc., Providence, RI, 1996, 91-101.

[27] F. D. Tall, On the cardinality of Lindelöf spaces with points $G_{\delta}$, Topology Appl. 63 (1995), no. 1, 21-38.

[28] M. Tkachenko, Chains and cardinals, Dokl. Akad. Nauk SSSR 239 (1978), 546-549 (in Russian).

[29] S. Todorčević, Some consequences of $M A+\neg w K H$, Topology Appl. 12 (1981), 187202.

[30] - , A note on the proper forcing axiom, in: Axiomatic Set Theory (Boulder, CO, 1983), Amer. Math. Soc., Providence, RI, 1984, 209-218.

Departamento de Matemática

Universidade de São Paulo

Caixa Postal 66281

São Paulo, SP

CEP: 05315-970, Brasil

E-mail: lucia@ime.usp.br

piotr@ime.usp.br

Received 10 July 1999;

in revised form 28 December 2000 